\title{
Accurate pricing of swaptions via Lower Bound
}

\author{
Anna Maria Gambaro* \\ Ruggero Caldana ${ }^{\dagger}$ \\ Gianluca Fusai ${ }^{\ddagger}$
}

\begin{abstract}
We propose a new lower bound for pricing European-style swaptions for a wide class of interest rate models. This method is applicable whenever the joint characteristic function of the state variables is either known in closed form or can be obtained numerically via some efficient procedure. Our lower bound involves the computation of a one dimensional Fourier transform independently of the underlying swap length. Finally the bound can be used as a control variate to reduce the confidence interval in the Monte Carlo simulation. We test our bound on different affine models, also allowing for jumps. The lower bound is found to be accurate and computationally efficient.
\end{abstract}

\footnotetext{
*Dipartimento di Statistica e Metodi Quantitativi, Università Milano Bicocca, Milano, Italy; Email: a.gambaro@campus.unimib.it

$\dagger$ Accenture, Milano, Italy; Email: ruggero.caldana@gmail.con

${ }^{\ddagger}$ Faculty of Finance, Cass Business School, City University London, UK and Dipartimento di Studi per l'Economia e l'Impresa, Università del Piemonte Orientale "A. Avogadro", Novara, Italy; Email: Gianluca.Fusai.1@city.ac.uk
} 


\section{Introduction}

Libor based derivatives (swaps, caps, swaptions) are the most liquid derivatives traded in financial markets. In particular a European swaption is a contract that gives the right to its owner to enter into an underlying interest rate swap, i.e it is an European option on a swap rate. It can be equivalently interpreted as an option on a portfolio of zero coupon bonds.

Let $t$ be the current date, $T$ be the option expiration date, $T_{1}, \ldots, T_{n}$ be the underlying swap payment dates (by construction $t<T<T_{1}<\ldots<T_{n}$ ) and $R$ the fixed rate of the swap. The payoff of a receiver swaption is

$$
\text { payoff }=\left(\sum_{h=1}^{n} w_{h} P\left(T, T_{h}\right)-1\right)^{+}
$$

where $w_{h}=R \cdot\left(T_{h}-T_{h-1}\right)$ for $h=1, . ., n-1$ and $w_{n}=R \cdot\left(T_{n}-T_{n-1}\right)+1, P\left(T, T_{h}\right)$ is the price at time $T$ of a zero coupon bond expiring at time $T_{h}$. The no-arbitrage fair price at time $\mathrm{t}$ is the discounted risk neutral expected value of the payoff

$$
C\left(t, T,\left\{T_{h}\right\}_{h=1}^{n}, R\right)=\mathbb{E}_{t}\left[e^{-\int_{t}^{T} r(\mathbf{X}(s)) d s}\left(\sum_{h=1}^{n} w_{h} P\left(T, T_{h}\right)-1\right)^{+}\right]
$$

where $r(\mathbf{X}(s))$ is the short rate at time $s$, and $\mathbf{X}(s)$ denotes the state vector at time $s$ of a multi-factor stochastic model.

Due to their importance and popularity, swaption market quotations are often used for calibration of interest rate models. However calibration procedure involves the pricing of a large number of swaptions (different option maturity, swap tenors and strikes), so the availability of an efficient pricing algorithm is required. Moreover Basel III accords introduced the Credit Value Adjustment (CVA) charge for over the counter contracts. ${ }^{1}$ It is interesting to note that for the most simple and popular kind of interest rate derivative, i.e. interest rate swap, the (unilateral) C.V.A. can be estimated as a portfolio of forward start European swaptions. $^{2}$

Since a closed-form formula of swaption price does not exist for many popular interest rate models, then several approximated pricing method have been developed in literature specially for affine interest rate models. The most important are those of Munk (1999), Collin-Dufresne and Goldstein (2002), Singleton and Umantsev (2002) and Schrager and Pelsser (2006).

Munk approximates the price of an option on a coupon bond by a multiple of the price of an option on a zero-coupon bond with time to maturity equal to the stochastic duration of the coupon bond. This method is fast but not very accurate for out of the money options.

The method of Collin-Dufresne and Goldstein is based on a Edgeworth expansion of the density of the swap rate and requires the calculation of the moments of the coupon bond. This procedure can be very time consuming.

Singleton and Umantsev (2002) introduce the idea of approximating the exercise region in the space of the state variables. This method has the advantage of producing precise results for a wide range of strikes (in particular for out of the money swaptions); however, it does not admit a simple extension to general affine interest rate models, because it requires the knowledge in closed form of the joint probability density function of the state variables. Moreover the Singleton and Umantsev method requires the calculus of as many Fourier inversion as the number of payment dates of the underlying swap contract. Hence the run time algorithm increases with the swap length.

${ }^{1} \mathrm{CVA}$ of a contract price $\mathrm{C}(\mathrm{t})$ is the risk neutral expectation of the loss

$$
C V A(t):=L D G \int_{0}^{T} \mathbb{E}\left[e^{-\int_{0}^{t} r(s) d s} \max (C(t), 0)\right] d \mathbb{P}_{D}(t),
$$

where $L G D$ is the Loss Given Default and $\mathbb{P}_{D}(t)$ is the default probability in the interval $(t, t+d t)$. Risk adjusted price is: $C(t)-C V A(t)$.

${ }^{2}$ C.V.A. of an interest rate swap with payment dates $T_{1}, \ldots, T_{n}$ and fixed rate $\mathrm{R}$, can be approximated by the following portfolio of swaptions:

$$
C V A(t) \simeq L G D \sum_{i=1}^{n} \mathbb{P}_{D}\left(T_{i-1}, T_{i}\right) \cdot S W O\left(t,\left\{T_{h}\right\}_{i-1}^{n}, R\right)
$$


Similarly to Singleton and Umantsev (2002), we propose a lower bound which is based on an approximation of the exercise region via an event set defined through a function of the model factors

$$
\mathcal{G}=\{\omega \in \Omega: g(\mathbf{X}(\omega), T) \geq k\}
$$

where $\mathbf{X}$ is the vector of the model factors, $\Omega$ is the state space and $g$ is a suitably chosen function approximating the exercise boundary.

Our pricing formula consists in the valuation of the option on the approximate exercise region and requires a single Fourier transform, performed with respect to the parameter $k$ in formula 1.2.

The approximation we propose has several advantages. First of all it is a lower bound, so the direction of the error is known a priori, further it is very general as it can be applied to a wide class of models, provided that the characteristic function of the state variates is known (explicitly or numerically). It involves the computation of only one Fourier inversion, independently of the number of cash flows of the underlying swap. Finally, it can be used as a control variate to improve the accuracy of the Monte Carlo simulation method.

The paper is organized as follows. Section 2 introduces a general formula for lower bound on swaption prices, based on an approximation of the exercise region. Then we apply the general lower bound formula to the case of affine interest rate models and we find an efficient algorithm to calculate analytically the approximated swaption price. Section 3 describes the approximate exercise set defined by the logarithm of the ZCBs portfolio geometric mean. Section 4 shows the results of numerical tests. Conclusive remarks are presented in last section.

\section{A Lower Bound on swaption prices}

In this section, we derive a general formula for lower bound on swaption prices. The price formula (1.1), once a change of measure to the T-forward measure is used, becomes

$$
\begin{aligned}
C\left(t, T,\left\{T_{h}\right\}_{h=1}^{n}, R\right) & =P(t, T) \cdot \mathbb{E}_{t}^{T}\left[\left(\sum_{h=1}^{n} w_{h} P\left(T, T_{h}\right)-1\right)^{+}\right] \\
& =P(t, T) \cdot \mathbb{E}_{t}^{T}\left[\left(\sum_{h=1}^{n} w_{h} P\left(T, T_{h}\right)-1\right) I(\mathcal{A})\right],
\end{aligned}
$$

where $I$ is the indicator function and $\mathcal{A}$ is the exercise region seen as a subset of the space events $\Omega$

$$
\mathcal{A}=\left\{\omega \in \Omega: \sum_{h=1}^{n} w_{h} P\left(T, T_{h}\right) \geq 1\right\} .
$$

Indeed, we observe that for any event set $\mathcal{G} \subset \Omega$

$$
\begin{aligned}
& \mathbb{E}_{t}^{T}\left[\left(\sum_{h=1}^{n} w_{h} P\left(T, T_{h}\right)-1\right)^{+}\right] \geq \mathbb{E}_{t}^{T}\left[\left(\sum_{h=1}^{n} w_{h} P\left(T, T_{h}\right)-1\right)^{+} I(\mathcal{G})\right] \\
& \geq \mathbb{E}_{t}^{T}\left[\left(\sum_{h=1}^{n} w_{h} P\left(T, T_{h}\right)-1\right) I(\mathcal{G})\right] .
\end{aligned}
$$

Then by discounting we obtain

$$
C\left(t, T,\left\{T_{h}\right\}_{h=1}^{n}, R\right) \geq L B(\mathcal{G}):=P(t, T) \cdot \mathbb{E}_{t}^{T}\left[\left(\sum_{h=1}^{n} w_{h} P\left(T, T_{h}\right)-1\right) I(\mathcal{G})\right]
$$

i.e. $L B(\mathcal{G})$ is a lower bound to the swaption price for all possible sets $\mathcal{G}$.

\section{$2.1 \quad$ Affine models}

For affine interest rate models the price at $T$ of a zero coupon bond with expiration date $T_{h}$ can be written as the exponential of a linear combination of the state variables

$$
P\left(T, T_{h}\right)=e^{\sum_{j=1}^{d} b_{h, j} X_{j}(T)+a_{h}}=e^{\mathbf{b}_{h}^{\top} \mathbf{X}(T)+a_{h}},
$$


where $a_{h}=A\left(T_{h}-T\right)$ and $\mathbf{b}_{h}=\mathbf{B}\left(T_{h}-T\right)$ are functions of the payment date $T_{h}$ and are typical of each model. We know from Duffie and Kan (1996) and Duffie, Pan and Singleton (2000) that under certain regularity conditions, the functions $A(\tau)$ and $\mathbf{B}(\tau)$ are the solution of a system of $d+1$ ordinary differential equations that are completely determined by the specification of the risk-neutral dynamics of the short rate. These equations can be solved through numerical integration starting from the initial conditions $A(0)=0, \mathbf{B}(0)=\underline{0}$ and the solutions are known in closed form for most common models.

Moreover from Duffie, Pan and Singleton (2000), we know that for affine models the risk neutral expected value of an exponential payoff has the form

$$
\mathbb{E}_{t}\left[e^{-\int_{t}^{T} r(\mathbf{X}(u)) d u} e^{i \boldsymbol{\lambda}^{\top} \mathbf{X}(s)}\right]=e^{\tilde{A}(s-t, T-s, \boldsymbol{\lambda})+\tilde{\mathbf{B}}(s-t, T-s, \boldsymbol{\lambda})^{\top} \mathbf{X}(t)},
$$

where $\mathbf{X}$ and $\boldsymbol{\lambda}$ are in $\mathbb{R}^{d}$ and the functions $\tilde{A}(\tau, T-s, \boldsymbol{\lambda})$ and $\tilde{\mathbf{B}}(\tau, T-s, \boldsymbol{\lambda})$ are solutions of the same ODE system of the zero coupon bond case, but with different initial conditions $(\tilde{A}(0, T-s, \boldsymbol{\lambda})=A(T-s)$ and $\tilde{\mathbf{B}}(0, T-s, \boldsymbol{\lambda})=i \boldsymbol{\lambda}+\mathbf{B}(T-s))$. Then the T-forward characteristic function of the model factors $\mathbf{X}$ can be obtained by

$$
\begin{aligned}
\mathbb{E}_{t}^{T}\left[e^{i \boldsymbol{\lambda}^{\top} \mathbf{X}(s)}\right] & =\frac{1}{P(t, T)} \mathbb{E}_{t}\left[e^{-\int_{t}^{T} r(\mathbf{X}(u)) d u} e^{i \boldsymbol{\lambda}^{\top} \mathbf{X}(s)}\right] \\
& =e^{\tilde{A}(s-t, T-s, \boldsymbol{\lambda})-A(T-t)+\left(\tilde{\mathbf{B}}(s-t, T-t, \boldsymbol{\lambda})^{\top}-\mathbf{B}(T-t)^{\top}\right) \mathbf{X}(t)} .
\end{aligned}
$$

Since we are interested in the case $s=T$ (forward measure at expiry date of the option), then we define the function

$$
\Phi(\boldsymbol{\lambda})=\mathbb{E}_{t}^{T}\left[e^{i \boldsymbol{\lambda}^{\top} \mathbf{X}(T)}\right]=e^{\tilde{A}(T-t, \boldsymbol{\lambda})-A(T-t)+\left(\tilde{\mathbf{B}}(T-t, \boldsymbol{\lambda})^{\top}-\mathbf{B}(T-t)^{\top}\right) \mathbf{X}(t)},
$$

where $\tilde{A}(\tau, \boldsymbol{\lambda})=\tilde{A}(\tau, 0, \boldsymbol{\lambda})$ and $\tilde{\mathbf{B}}(\tau, \boldsymbol{\lambda})=\tilde{\mathbf{B}}(\tau, 0, \boldsymbol{\lambda})$.

We define the set $\mathcal{G}$ using a linear function of the state variates

$$
\mathcal{G}=\{\omega \in \Omega: g(\mathbf{X}(T)) \geq k\}=\left\{\omega \in \Omega: \boldsymbol{\beta}^{\top} \mathbf{X}(T)+\alpha \geq k\right\},
$$

where $\boldsymbol{\beta}$ is a constant vector of length $d, \alpha \in \mathbb{R}$ and $k$ is a free parameter. $k$ can be chosen such that it optimizes the value of the lower bound.

Proposition 1. The lower bound to the European swaption price, for affine interest rate models, is given by the following formula

$$
\widehat{L B}\left(t, T,\left\{T_{h}\right\}_{h=1}^{n}, R\right)=\max _{k \in \mathbb{R}} L B\left(k ; t, T,\left\{T_{h}\right\}_{h=1}^{n}, R\right),
$$

where

$$
L B\left(k ; t, T,\left\{T_{h}\right\}_{h=1}^{n}, R\right)=P(t, T) \frac{e^{-\delta k}}{\pi} \int_{0}^{+\infty} e^{-i \gamma k} \psi_{\delta}(\gamma) d \gamma,
$$

and

$$
\psi_{\delta}(\gamma)=\left(\sum_{h=1}^{n} w_{h} e^{a_{h}} \Phi\left(-i \mathbf{b}_{h}+(\gamma-i \delta) \boldsymbol{\beta}\right)-\Phi((\gamma-i \delta) \boldsymbol{\beta})\right) \frac{e^{(i \gamma+\delta) \alpha}}{i \gamma+\delta},
$$

where $\delta$ is a positive constant.

Proof: See Appendix (A.1).

\section{The geometric average approximate exercise region}

The approximate exercise set is defined through the logarithm of the geometric average of the portfolio of zero coupon bonds

$$
\begin{aligned}
\mathcal{G} & =\{\omega \in \Omega: g(\mathbf{X}(T)) \geq k\}, \\
G(\mathbf{X}(T)) & =\prod_{h=1}^{n} P\left(T, T_{h}\right)^{w_{h}}, \\
g(\mathbf{X}(T)) & =\ln (G(\mathbf{X}(T)))=\sum_{h=1}^{n} w_{h} \ln \left(P\left(T, T_{h}\right)\right) .
\end{aligned}
$$


In particular $\mathcal{G}$ and $g(\mathbf{X})$ are given by

$$
\mathcal{G}=\left\{\omega \in \Omega: \boldsymbol{\beta}^{\top} \mathbf{X}(T)+\alpha \geq k\right\},
$$

where $P\left(T, T_{h}\right)=e^{\mathbf{b}_{h} \top \mathbf{X}(T)+a_{h}}, \boldsymbol{\beta}=\sum_{h=1}^{n} w_{h} \mathbf{b}_{h}$ and $\alpha=\sum_{h=1}^{n} w_{h} a_{h}$.

Since we don't know the optimum value of the parameter $k$, then the pricing method requires the maximization of the lower bound, $L B\left(k ; t, T,\left\{T_{h}\right\}_{h=1}^{n}, R\right)$, seen as a function of $k$.

The optimization can be accelerated looking for a good starting point. We suggest the following

$$
\tilde{k}=\log \left(\frac{1}{\sum_{h=1}^{n} w_{h}}\right)=-\log \left(\sum_{h=1}^{n} R\left(T_{h}-T_{h-1}\right)\right) .
$$

According to this choice $\mathcal{G}_{\tilde{k}}=\{\omega \in \Omega: g(\mathbf{X}(T)) \geq \tilde{k}\}$ is the greatest possible subset of the true exercise region, $\mathcal{A}$.

In fact normalizing the weights, the expression of the true exercise region can be rewritten as

$$
\begin{aligned}
& \mathcal{A}=\left\{\omega \in \Omega: \sum_{h=1}^{n} w_{h} P\left(T, T_{h}\right) \geq 1\right\}=\left\{\omega \in \Omega: \sum_{h=1}^{n} \tilde{w}_{h} P\left(T, T_{h}\right) \geq e^{\tilde{k}}\right\}= \\
& =\left\{\omega \in \Omega: A(\mathbf{X}) \geq e^{\tilde{k}}\right\}
\end{aligned}
$$

where $A(\mathbf{X})$ is the arithmetic mean of the ZCBs portfolio, $\tilde{w}_{h}=\frac{w_{h}}{\sum_{h=1}^{n} w_{h}}$ and so $\sum_{h=1}^{n} \tilde{w}_{h}=1$.

By the arithmetic-geometric inequality we know that $A(\mathbf{X}) \geq G(\mathbf{X}) \forall \mathbf{X}$, then $\forall k>\tilde{k}$

$$
\mathcal{A} \supseteq \mathcal{G}_{\tilde{k}} \supseteq \mathcal{G}_{k} .
$$

Instead if $k<\tilde{k}$ then it is no more guaranteed that $\mathcal{G}_{k}$ is a subset of the true exercise region.

\section{Models and Numerical results}

This section presents the examined models and discuss the numerical results.

\subsection{Affine Gaussian models}

Affine Gaussian models assign the following stochastic differential equation (S.D.E.) to the state variable $\mathrm{X}$

$$
\begin{aligned}
& d \mathbf{X}(t)=K(\boldsymbol{\theta}-\mathbf{X}(t)) d t+\Sigma d \mathbf{W}(t), \\
& \mathbf{X}(0)=\mathbf{x}_{0},
\end{aligned}
$$

where $\mathbf{W}_{t}$ is a standard $d$-dimensional Brownian motion, $K$ is a $d \times d$ diagonal matrix and $\Sigma$ is a $d \times d$ triangular matrix. The short rate is obtained as a linear combination of the state vector $\mathbf{X}$; it is always possible to rescale the components $X_{i}(t)$ and assume that

$$
r(t)=\phi+\sum_{i=1}^{d} X_{i}(t)
$$

without loss of generality $(\phi \in \mathbb{R})$.

The T-forward characteristic function of $\mathbf{X}$ is

$$
\Phi(\boldsymbol{\lambda})=\mathbb{E}_{t}^{T}\left[e^{i \boldsymbol{\lambda}^{\top} \mathbf{X}(T)}\right]=e^{i \boldsymbol{\lambda}^{\top} \boldsymbol{\mu}(t, T)-\frac{1}{2} \boldsymbol{\lambda}^{\top} V(t, T) \boldsymbol{\lambda}},
$$

where $\boldsymbol{\mu}$ is the T-forward expected value and $V$ is the covariance matrix (assuming $\mathrm{K}$ is diagonal):

$$
\begin{aligned}
\boldsymbol{\mu}(t, s) & =\mathbb{E}_{t}^{T}[\mathbf{X}(s)]=\mathbb{E}_{t}[\mathbf{X}(s)]-\left(I_{n \times n}-e^{-K(s-t)}\right) K^{-1} \Sigma \Sigma^{\top}\left(K^{-1}\right)^{\top} \mathbf{g}-V e^{-K^{\top}(T-s)}\left(K^{-1}\right)^{\top} \mathbf{g} \\
\mathbb{E}_{t}[\mathbf{X}(s)] & =e^{-K(s-t)}(X(t)-\boldsymbol{\theta})+\boldsymbol{\theta} \\
V_{i j}(t, s) & =\left(\Sigma \Sigma^{\top}\right)_{i j}\left(\frac{1-e^{-\left(K_{i i}+K_{j j}\right)(s-t)}}{K_{i i}+K_{j j}}\right) .
\end{aligned}
$$


where $\mathbf{g}=[1,1,1, \ldots, 1]^{\top}$ is a column vector of length $\mathrm{d}$.

For this type of process, the lower bound can be calculated analytically

$$
L B\left(k ; t, T,\left\{T_{h}\right\}_{h=1}^{n}, R\right)=P(t, T)\left(\sum_{h=1}^{n} w_{h} e^{a_{h}+\mathbf{b}_{h}^{\top} \boldsymbol{\mu}+\frac{1}{2} V_{h}+\frac{1}{2} d_{h}^{2}} N\left(d_{h}-d\right)-N(-d)\right),
$$

where

$$
\begin{aligned}
d & =\frac{k-\boldsymbol{\beta}^{\top} \boldsymbol{\mu}-\alpha}{\sqrt{\boldsymbol{\beta}^{\top} V \boldsymbol{\beta}}}, \\
d_{h} & =\mathbf{b}_{h}^{\top} \mathbf{v} \\
V_{h} & =\mathbf{b}_{h}^{\top}\left(V-\mathbf{v} \mathbf{v}^{\top}\right) \mathbf{b}_{h}, \\
\mathbf{v} & =\frac{V \boldsymbol{\beta}}{\sqrt{\boldsymbol{\beta}^{\top} V \boldsymbol{\beta}}}
\end{aligned}
$$

and $N(x)=\frac{1}{\sqrt{2 \pi}} \int_{-\infty}^{x} e^{-\frac{y^{2}}{2}} d y$ is the cumulative distribution function of a standard normal. See details in Appendix (A.2).

\subsection{Multi-factor Cox-Ingersoll-Ross (CIR) model}

The state vector of the model evolves according to the following system of SDE

$$
\begin{aligned}
d X_{i}(t) & =a_{i}\left(\theta_{i}-X_{i}(t)\right) d t+\sigma_{i} \sqrt{X_{i}(t)} d W^{i}(t), \\
\mathbf{X}(0) & =\mathbf{x}_{0}
\end{aligned}
$$

where $i=1, \ldots, d, W^{i}(t)$ are independent standard Brownian motions, $a_{i}, \theta_{i}$ and $\sigma_{i}$ are positive constants. The short rate is obtained by

$$
r(t)=\phi+\sum_{i=1}^{d} X_{i}(t)
$$

where $\phi \in \mathbb{R}$.

Using the results in Collin-Dufresne and Goldstein (2002), we can calculate the zero coupon bond price and the T-forward characteristic function. In particular we deduce the functions $A(\tau), \mathbf{B}(\tau), \tilde{A}(\tau, \boldsymbol{\lambda})$ and $\tilde{\mathbf{B}}(\tau, \boldsymbol{\lambda})$ in formula $(2.4)$,

$$
\begin{aligned}
A(\tau) & =-\phi \tau+\sum_{j=1}^{d}\left[\frac{2 a_{j} \theta_{i}}{h_{j}-a_{j}} \tau-\frac{2 a_{j} \theta_{j}}{\sigma_{j}^{2}} \ln \left(\frac{\left(a_{j}+h_{j}\right)\left(e^{h_{j} \tau}-1\right)+2 h_{j}}{2 h_{j}}\right)\right], \\
B_{j}(\tau) & =\frac{-2\left(e^{h_{j} \tau}-1\right)}{\left(a_{j}+h_{j}\right)\left(e^{h_{j} \tau}-1\right)+2 h_{j}}, \\
\tilde{A}(\tau, \boldsymbol{\lambda}) & =-\phi \tau+\sum_{j=1}^{d}\left[\frac{2 a_{j} \theta_{i}}{h_{j}-a_{j}} \tau-\frac{2 a_{j} \theta_{j}}{\sigma_{j}^{2}} \ln \left(\frac{\sigma_{j}^{2}\left(\left(i \lambda_{j}+\mu_{j}^{+}\right)-\left(i \lambda+\mu_{j}^{-}\right) e^{h_{j} \tau}\right)}{2 h_{j}}\right)\right], \\
\tilde{B}_{j}(\tau, \boldsymbol{\lambda}) & =\frac{i \lambda_{j}\left(\mu_{j}^{-}-\mu_{j}^{+} e^{h_{j} \tau}\right)+\frac{2\left(e^{h_{j} \tau}-1\right)}{\sigma_{j}^{2}}}{i \lambda_{j}\left(e^{h_{j} \tau}-1\right)+\left(\mu_{j}^{-} e^{h_{j} \tau}-\mu_{j}^{+}\right)},
\end{aligned}
$$

where $h_{j}=\sqrt{a_{j}^{2}+2 \sigma_{j}^{2}}$ and $\mu_{j}^{ \pm}=\frac{-a_{j} \pm h_{j}}{\sigma_{j}^{2}}$.

\subsection{Gaussian model with double Exponential Jumps}

The vector of model factors evolves according to the following S.D.E.

$$
\begin{aligned}
& d \mathbf{X}(t)=K(\boldsymbol{\theta}-\mathbf{X}(t)) d t+\Sigma d \mathbf{W}(t)+d \mathbf{Z}^{+}(t)-d \mathbf{Z}^{-}(t), \\
& \mathbf{X}(0)=\mathbf{x}_{0},
\end{aligned}
$$


where $\mathbf{W}_{t}$ is a standard $d$-dimensional Brownian motion, $K$ is a $d \times d$ diagonal matrix, $\Sigma$ is a $d \times d$ triangular matrix and $\mathbf{Z}^{ \pm}$are pure jumps processes whose jumps have fixed probability distribution $\nu$ on $\mathbb{R}^{d}$ and constant intensity $\mu^{ \pm}$. The short rate is obtained as a linear combination of the state vector $\mathbf{X}$. In particular $\mathbf{Z}^{ \pm}$are compound Poisson processes with jump size having exponential distribution

$$
Z_{l}^{ \pm}=\sum_{j=1}^{N^{ \pm}(t)} Y_{j, l}^{ \pm},
$$

where $l=1, \ldots, d$ is the factor index, $N^{ \pm}(t)$ are Poisson process with intensity $\frac{\mu^{ \pm}}{d} 3$ and $Y_{j, l}^{ \pm}$, for a fixed $l$, are independent identically distributed exponential random variables of mean parameters $m_{l}^{ \pm}$:

$$
Y_{j, l}^{ \pm} \sim \nu\left(m_{l}^{ \pm}\right)=\frac{1}{m_{l}^{ \pm}} \exp \left(\frac{y}{m_{l}^{ \pm}}\right) .
$$

Since $\mu^{ \pm}$do not depend on $\mathbf{X}$, we know from Duffie, Pan and Singleton (2000) that: (a) the functions $\mathbf{B}(\tau)$ and $\tilde{\mathbf{B}}(\tau, \boldsymbol{\lambda})$ in formula (2.4) are independent of jumps and they are calculated as in affine Gaussian model case; (b)the functions $A(\tau)$ and $\tilde{A}(\tau, \boldsymbol{\lambda})$ in formula (2.4) are sum of two components, diffusive and jump: $A(\tau)=A^{D}(\tau)+A^{J}(\tau)$ and $\tilde{A}(\tau, \boldsymbol{\lambda})=\tilde{A}^{D}(\tau, \boldsymbol{\lambda})+\tilde{A}^{J}(\tau, \boldsymbol{\lambda})$. The diffusive part can be obtained as in affine Gaussian model case.

Then we obtain that

$$
\Phi(\boldsymbol{\lambda})=\mathbb{E}_{t}^{T}\left[e^{i \boldsymbol{\lambda}^{\top} \mathbf{X}(T)}\right]=\Phi^{D}(\boldsymbol{\lambda}) e^{\tilde{A}^{J}(T-t, \boldsymbol{\lambda})-A^{J}(T-t)},
$$

where $\Phi^{D}(\boldsymbol{\lambda})$ is the T-forward characteristic function of affine Gaussian model. The jumps component is calculated using the characteristic function of the jumps size distribution $\nu$

$$
\begin{aligned}
& \theta^{ \pm}(\mathbf{c})=\int_{\mathbb{R}^{d}} e^{\mathbf{c}^{\top} \mathbf{y}} d \nu(\mathbf{y})=\frac{1}{d} \sum_{j=1}^{d} \frac{1}{1-m_{j}^{ \pm} c_{j}}=\frac{1}{d} \sum_{j=1}^{d} \frac{\eta_{j}^{ \pm}}{\eta_{j}^{ \pm}-c_{j}}, \\
& \tilde{A}^{J}(T-t, \boldsymbol{\lambda})=\mu^{+} \int_{t}^{T} d s\left(\theta^{+}(\tilde{\mathbf{B}}(s, \boldsymbol{\lambda}))-1\right)+\mu^{-} \int_{t}^{T} d s\left(\theta^{-}(\tilde{\mathbf{B}}(s, \boldsymbol{\lambda}))-1\right), \\
& A^{J}(T-t)=\tilde{A}^{J}(T-t, \mathbf{0}),
\end{aligned}
$$

where $c \in \mathbb{C}^{d}$ and $\eta_{j}^{ \pm}=\frac{1}{m_{j}^{ \pm}}$. The function $\tilde{A}^{J}(\tau, \boldsymbol{\lambda})$ is available in closed form

$$
\begin{aligned}
\tilde{A}^{J}(\tau, \boldsymbol{\lambda}) & =\frac{\mu^{+}}{d} \sum_{j=1}^{d} \frac{-\tau}{1+\eta_{j}^{+} K_{j j}}+\frac{\eta_{j}^{+}}{1+\eta_{j}^{+} K_{j j}} \log \left(\frac{\left(1+i \lambda_{j} K_{j j}\right) e^{-K_{j j} \tau}-1-\eta_{j}^{+} K_{j j}}{K_{j j}\left(i \lambda_{j}-\eta_{j}^{+}\right)}\right) \\
& +\frac{\mu^{-}}{d} \sum_{j=1}^{d} \frac{-\tau}{1-\eta_{j}^{-} K_{j j}}-\frac{\eta_{j}^{-}}{1-\eta_{j}^{-} K_{j j}} \log \left(\frac{\left(1+i \lambda_{j} K_{j j}\right) e^{-K_{j j} \tau}-1+\eta_{j}^{-} K_{j j}}{K_{j j}\left(i \lambda_{j}+\eta_{j}^{-}\right)}\right) .
\end{aligned}
$$

\subsection{Balduzzi, Das, Foresi and Sundaram model}

In the model proposed in Balduzzi, Das, Foresi and Sundaram (1996), the interest rate follows the same stochastic process as in CIR model, but the long-mean $\theta(t)$ and the variance $V(t)$ are stochastic, according to the following system of SDEs

$$
\left\{\begin{array}{l}
d r(t)=k(\theta(t)-r(t)) d t+\sqrt{V(t)} d W(t) \\
d \theta(t)=\alpha(\beta-\theta(t)) d t+\eta d Z(t) \\
d V(t)=a(b-V(t)) d t+\phi \sqrt{V(t)} d Y(t) \\
d W(t) d Y(t)=\rho d t \\
r(0)=r_{0}, \theta(0)=\theta_{0} \text { and } V(0)=v_{0}
\end{array}\right.
$$

$k, \alpha, \beta, \eta, a, b$ and $\phi$ are positive constants and $\rho \in[-1,1]$. In order to align the notation with previous sections we denote $\mathbf{X}(t)=[r(t), \theta(t), V(t)]^{\top}$. In this model the characteristic function of the state vector

\footnotetext{
${ }^{3} N^{ \pm}(t)$ represent the number of positive or negative jumps before the time $t$
} 
$\mathbf{X}$ can not be obtained in closed form ${ }^{4}$, but can be calculated numerically, solving the following system of ordinary differential equations

$$
\begin{aligned}
\frac{d \tilde{A}(\tau, \boldsymbol{\lambda})}{d \tau} & =-\alpha \beta \tilde{B}_{2}(\tau, \boldsymbol{\lambda})+\frac{1}{2} \eta^{2} \tilde{B}_{2}(\tau, \boldsymbol{\lambda})^{2}-a b \tilde{B}_{3}(\tau, \boldsymbol{\lambda}), \\
\tilde{B}_{1}(\tau, \boldsymbol{\lambda}) & =\lambda_{1} e^{-k \tau}-\frac{1-e^{-k \tau}}{k}, \\
\tilde{B}_{2}(\tau, \boldsymbol{\lambda}) & =\left(k \lambda_{1}+1\right) \frac{e^{-k \tau}-e^{-\alpha \tau}}{\alpha-k}+\lambda_{2} e^{-\alpha \tau}-\frac{1-e^{-\alpha \tau}}{\alpha}, \\
\frac{d \tilde{B}_{3}(\tau, \boldsymbol{\lambda})}{d \tau} & =\frac{1}{2} \tilde{B}_{1}(\tau, \boldsymbol{\lambda})^{2}+a \tilde{B}_{3}(\tau, \boldsymbol{\lambda})+\frac{1}{2} \phi^{2} \tilde{B}_{3}(\tau, \boldsymbol{\lambda})^{2}+\rho \phi \tilde{B}_{1}(\tau, \boldsymbol{\lambda}) \tilde{B}_{3}(\tau, \boldsymbol{\lambda}) .
\end{aligned}
$$

The functions $A(\tau)$ and $\mathbf{B}(\tau)$ (i.e. the zero coupon bond price) can be obtained solving the previous system and setting $\boldsymbol{\lambda}=\underline{0}$.

\subsection{Numerical Results}

Apart from the Vasicek model for which a simple closed form solution is available, Monte Carlo is used as benchmark for the computation of the true swaption price. The $97.5 \%$ mean-centered Monte Carlo Confidence Interval ${ }^{5}$ is used as measure of the accuracy of the Monte Carlo method. Simulation of the affine 3-factor Gaussian and 2-factor CIR models is implemented by sampling from the exact distribution. The Gaussian with jumps model and the BDFS model are simulated using an Euler-Maruyama scheme with a time step of 0.0005 . The number of simulations is chosen depending on the complexity of the model and it is specified in each table caption. Antithetic variates technique is also used for the affine 3 -factor Gaussian model and the BDFS model.

For the Vasicek model and the 3-factor Gaussian model, lower bound is obtained via the closed formula described in section (4.1). For the 2-factor CIR model and the Gaussian with jumps model, the integrals involved in the lower bound are evaluated by a Gauss-Kronrod quadrature rule, using Matlab's built-in function quadgk. For the BDFS model the system of ordinary differential equations is solved numerically using the Matlab function ode45 based on the Dormand-Prince method. Due to the complexity of the problem we adopt a Gauss-Legendre quadrature rule. The calculus of the lower bound with a geometric mean region $\mathcal{G}$ involves the optimization of the function $L B\left(k ; t, T,\left\{T_{h}\right\}_{h=1}^{n}, R\right)$ with respect to the parameter $k$. The optimization is performed via Matlab functions fminunc.

Another important fact is that our lower bound formula is very suitable to be used as a control variate to reduce Monte Carlo error. The approximated formula is easy to implement in a Monte Carlo scheme and turns out to be very effective (see Caldana, Fusai, Gnoatto and Grasselli (2014) for details).

Swaption prices for different tenor and maturities are reported in Tables (1-5) with the relative overall computing time for each pricing method.

Moreover, Figures (1-5) compare graphically the relative error of the three proposed pricing methods.

\subsubsection{Vasicek model, three-factors Gaussian model and Cox-Ingersoll and Ross model}

We verify the accuracy of our new lower bound using models and parameter values already examined in literature $^{6}$

- Vasicek model: $K=0.05, \theta=0.05, \Sigma=0.01, x_{0}=0.05$ and $\phi=0$;

- 3-factors Gaussian model: $K=\left[\begin{array}{ccc}1.0 & 0 & 0 \\ 0 & 0.2 & 0 \\ 0 & 0 & 0.5\end{array}\right], \theta=[0,0,0]^{\top}, \boldsymbol{\sigma}=[0.01,0.005,0.002]^{\top}$,

$$
\rho=\left[\begin{array}{ccc}
1 & -0.2 & -0.1 \\
-0.2 & 1 & 0.3 \\
-0.1 & 0.3 & 1
\end{array}\right], \Sigma=\operatorname{diag}(\boldsymbol{\sigma}) \cdot \operatorname{chol}(\rho)^{7}, x_{0}=[0.01,0.005,-0.02] \text { and } \phi=0.06 ;
$$

\footnotetext{
${ }^{4}$ A semi-analytical solution for functions $\tilde{A}(\tau, \boldsymbol{\lambda})$ and $\tilde{B}_{3}(\tau, \boldsymbol{\lambda})$ is available but it requires the evaluation of Kummer's functions of the first and second kind. Kummer's functions are not analytic but have series and integral representation. However we find that the numerical solution of the ODE system is much more efficient than the evaluation of the semi analytical form.

${ }^{5}$ Note that we use the quantile function of a Student's t distribution.

${ }^{6}$ Schrager and Pelsser (2006) and Duffie and Singleton (1997) for the 2-factors C.I.R. model

${ }^{7} \operatorname{diag}(\boldsymbol{\sigma})$ means the diagonalization of the vector $\boldsymbol{\sigma}$ and $\operatorname{chol}(\rho)$ means the Cholesky decomposition of the correlation matrix $\rho$, where $\boldsymbol{\sigma}$ and $\rho$ are the volatility vector and the correlation matrix, respectively, of the original paper.
} 
- 2-factors Cox-Ingersoll and Ross model: $\mathbf{a}=[0.5080,-0.0010]^{\top}, \boldsymbol{\theta}=[0.4005,-0.7740]^{\top}, \boldsymbol{\sigma}=$ $[0.023,0.019]^{\top}, x_{0}=[0.374,0.258]$ and $\phi=-0.58$.

Numerical results for these models are shown in Tables 1, 2 and 3.

\subsubsection{Two-factor Gaussian model with double exponential jumps}

We test the affine Gaussian with Jumps interest rate model using the following parameter values

- Gaussian parameters: $K=\left[\begin{array}{cc}0.5 & 0 \\ 0 & 0.2\end{array}\right], \theta=[0,0]^{\top}, \boldsymbol{\sigma}=[0.01,0.005]^{\top}$, $\rho=\left[\begin{array}{cc}1 & -0.2 \\ -0.2 & 1\end{array}\right], \Sigma=\operatorname{diag}(\boldsymbol{\sigma}) \cdot \operatorname{chol}(\rho), x_{0}=[0.01,0.005]$ and $\phi=0.005$

- Jumps parameters: $\boldsymbol{\mu}^{+}=0.001, \mathbf{m}^{+}=[0.01,0.01], \boldsymbol{\mu}^{-}=0.001, \mathbf{m}^{-}=[0.01,0.01]$.

Numerical results for this model are shown in table 4.

\subsubsection{Balduzzi, Das, Foresi and Sundaram model}

In order to prove the accuracy of our bounds for a wider class of models, we consider a stochastic volatility (and long run mean) model. We use the following parameter values, proposed in Balduzzi, Das, Foresi and Sundaram (1996) : $k=0.25, \alpha=0.76, \beta=0.12, \eta=0.02, a=0.29, b=0.0007, \phi=0.003$ and $\rho$ $=-0.12$.

Numerical results for this model are shown in Table 5.

\section{Conclusions}

This paper provides a new lower bound method for pricing of swaptions that is accurate, fast and applicable to a wide range of interest rate models. Our algorithm is particularly efficient because it requires the computation of only one Fourier inversion. Existing approximations (for instance Singleton and Umantsev method) require a number of Fourier inversions equal to the number of payment dates of the underlying swap. An approximate exercise region defined by the log-geometric mean of the ZCBs portfolio is tried out for the first time for swaption pricing. From the numerical tests we find that the approximation is much faster than Monte Carlo method and it is also very accurate across different maturities, tenors and strikes. Numerical results are presented across a wide class of models, including model with jumps and stochastic volatility. For all these models our lower bound is applicable and it is accurate, instead the Singleton and Umantsev method is not applicable if the density function is not known in analytical form. Moreover the lower bound is very effective as control variate to reduce the computation time and the error of the Monte Carlo. Hence, our model could be very suitable also for calibration purposes. 


\section{Vasicek model}

Payer Swaptions (ATMF)

\begin{tabular}{|c|cc|cc|cc|}
\hline Option mat. & \multicolumn{2}{|c|}{1} & \multicolumn{2}{c|}{2} & \multicolumn{2}{c|}{5} \\
\hline Swap length & LB & Jam. & LB & Jam. & LB & Jam. \\
\hline \multirow{2}{*}{1} & 35.670 & 35.670 & 46.836 & 46.836 & 59.501 & 59.501 \\
& $10^{-4}$ & & $10^{-4}$ & & $10^{-4}$ & \\
\hline \multirow{2}{*}{2} & 67.953 & 67.953 & 89.234 & 89.234 & 113.412 & 113.412 \\
& $10^{-4}$ & & $10^{-4}$ & & $10^{-4}$ & \\
\hline \multirow{2}{*}{5} & 147.645 & 147.645 & 193.957 & 193.957 & 246.875 & 246.875 \\
& $10^{-4}$ & & $10^{-4}$ & & $10^{-4}$ & \\
\hline \multirow{2}{*}{10} & 238.273 & 238.273 & 313.243 & 313.243 & 399.674 & 399.674 \\
& $10^{-4}$ & & $10^{-4}$ & & $10^{-4}$ & \\
\hline
\end{tabular}

Payer Swaptions (ITMF: $0.85 \times$ ATMF)

\begin{tabular}{|c|cc|cc|cc|}
\hline Option mat. & \multicolumn{2}{|c|}{1} & \multicolumn{2}{c|}{2} & \multicolumn{2}{c|}{5} \\
\hline Swap length & LB & Jam. & LB & Jam. & LB & Jam. \\
\hline \multirow{2}{*}{1} & 80.591 & 80.591 & 86.861 & 86.861 & 91.405 & 91.405 \\
& $10^{-4}$ & & $10^{-4}$ & & $10^{-4}$ & \\
\hline \multirow{2}{*}{2} & 155.872 & 155.872 & 167.452 & 167.452 & 175.622 & 175.622 \\
& $10^{-4}$ & & $10^{-4}$ & & $10^{-4}$ & \\
\hline \multirow{2}{*}{5} & 353.282 & 353.282 & 376.199 & 376.199 & 391.032 & 391.032 \\
& $10^{-4}$ & & $10^{-4}$ & & $10^{-4}$ & \\
\hline \multirow{2}{*}{10} & 605.661 & 605.661 & 637.301 & 637.301 & 654.439 & 654.439 \\
& $10^{-4}$ & & $10^{-4}$ & & $10^{-4}$ & \\
\hline
\end{tabular}

Payer Swaptions (OTMF: $1.15 \times$ ATMF)

\begin{tabular}{|c|cc|cc|cc|}
\hline Option mat. & \multicolumn{2}{|c|}{1} & \multicolumn{2}{c|}{2} & \multicolumn{2}{c|}{5} \\
\hline Swap length & LB & Jam. & LB & Jam. & LB & Jam. \\
\hline \multirow{2}{*}{1} & 11.247 & 11.247 & 21.169 & 21.169 & 35.825 & 35.825 \\
& $10^{-4}$ & & $10^{-4}$ & & $10^{-4}$ & \\
\hline \multirow{2}{*}{2} & 20.781 & 20.781 & 39.551 & 39.551 & 67.525 & 67.525 \\
& $10^{-4}$ & & $10^{-4}$ & & $10^{-4}$ & \\
\hline \multirow{2}{*}{5} & 41.394 & 41.394 & 81.330 & 81.330 & 142.400 & 142.400 \\
& $10^{-4}$ & & $10^{-4}$ & & $10^{-4}$ & \\
\hline \multirow{2}{*}{10} & 58.744 & 58.744 & 121.059 & 121.059 & 220.008 & 220.008 \\
& $10^{-4}$ & & $10^{-4}$ & & $10^{-4}$ & \\
\hline
\end{tabular}

Table 1: The three tables represent matrices of swaption prices at three different strikes for the Vasicek model. For each swaption we report on the first line the price in basis points and on the second line the error in basis points. The error of the lower bound is the difference from the exact Jamshidian method (Jam.). 


\section{Three-factor Gaussian model}

\begin{tabular}{|c|c|c|c|c|c|c|c|c|c|}
\hline \multicolumn{10}{|c|}{ Payer Swaptions (ATMF) } \\
\hline Opt. Mat. & \multicolumn{3}{|c|}{1} & \multicolumn{3}{|c|}{2} & \multicolumn{3}{|c|}{5} \\
\hline Swap length & $\mathrm{MC}$ & LB & $\mathrm{MC}(\mathrm{CV})$ & $\mathrm{MC}$ & LB & $\mathrm{MC}(\mathrm{CV})$ & $\mathrm{MC}$ & LB & $\mathrm{MC}(\mathrm{CV})$ \\
\hline 1 & $\begin{array}{c}20.814 \\
0.010\end{array}$ & 20.817 & $\begin{array}{c}20.817 \\
10^{-4}\end{array}$ & $\begin{array}{c}23.554 \\
0.011\end{array}$ & 23.554 & $\begin{array}{c}23.554 \\
10^{-4}\end{array}$ & $\begin{array}{c}23.217 \\
0.011\end{array}$ & 23.207 & $\begin{array}{c}23.207 \\
10^{-4}\end{array}$ \\
\hline 2 & $\begin{array}{c}33.114 \\
0.015\end{array}$ & 33.119 & $\begin{array}{c}33.119 \\
10^{-4}\end{array}$ & $\begin{array}{c}38.430 \\
0.018\end{array}$ & 38.434 & $\begin{array}{c}38.434 \\
10^{-4}\end{array}$ & $\begin{array}{c}38.728 \\
0.018\end{array}$ & 38.722 & $\begin{array}{c}38.722 \\
10^{-4}\end{array}$ \\
\hline 5 & $\begin{array}{c}53.325 \\
0.025\end{array}$ & 53.312 & $\begin{array}{c}53.312 \\
10^{-4}\end{array}$ & $\begin{array}{c}63.676 \\
0.029\end{array}$ & 63.686 & $\begin{array}{c}63.686 \\
10^{-4}\end{array}$ & $\begin{array}{c}65.673 \\
0.030\end{array}$ & 65.683 & $\begin{array}{c}65.683 \\
10^{-4}\end{array}$ \\
\hline 10 & $\begin{array}{c}65.583 \\
0.030\end{array}$ & 65.579 & $\begin{array}{c}65.583 \\
0.001\end{array}$ & $\begin{array}{c}79.090 \\
0.036\end{array}$ & 79.062 & $\begin{array}{c}79.067 \\
0.001\end{array}$ & $\begin{array}{c}82.164 \\
0.037\end{array}$ & 82.156 & $\begin{array}{c}82.159 \\
10^{-4}\end{array}$ \\
\hline $\begin{array}{c}\text { Overall } \\
\text { time }(\mathrm{sec})\end{array}$ & $\begin{array}{c}\mathrm{MC} \\
32.045\end{array}$ & $\begin{array}{c}\text { LB } \\
0.122\end{array}$ & $\begin{array}{c}\mathrm{MC}(\mathrm{CV}) \\
3.657\end{array}$ & & & & & & \\
\hline
\end{tabular}

Payer Swaptions (ITMF: $0.85 \times$ ATMF)

\begin{tabular}{|c|ccc|ccc|ccc|}
\hline Opt. Mat. & \multicolumn{3}{|c|}{1} & \multicolumn{3}{|c|}{2} & \multicolumn{3}{|c|}{5} \\
\hline Swap length & $\mathrm{MC}$ & $\mathrm{LB}$ & $\mathrm{MC}(\mathrm{CV})$ & $\mathrm{MC}$ & $\mathrm{LB}$ & $\mathrm{MC}(\mathrm{CV})$ & $\mathrm{MC}$ & $\mathrm{LB}$ & $\mathrm{MC}(\mathrm{CV})$ \\
\hline \multirow{2}{*}{1} & 79.446 & 79.445 & 79.445 & 78.404 & 78.404 & 78.404 & 69.446 & 69.442 & 69.442 \\
& 0.003 & & $10^{-4}$ & 0.005 & & $10^{-4}$ & 0.005 & $10^{-4}$ \\
\hline \multirow{2}{*}{2} & 154.563 & 154.563 & 154.563 & 150.909 & 150.911 & 150.911 & 131.95 & 131.949 & 131.949 \\
& 0.003 & & $10^{-4}$ & 0.005 & & $10^{-4}$ & 0.007 & $10^{-4}$ \\
\hline \multirow{2}{*}{5} & 361.470 & 361.469 & 361.469 & 346.275 & 346.275 & 346.275 & 295.162 & 295.162 & 295.162 \\
& 0.001 & & $10^{-4}$ & 0.003 & & $10^{-4}$ & 0.006 & $10^{-4}$ \\
\hline \multirow{2}{*}{10} & 636.982 & 636.982 & 636.982 & 604.809 & 604.81 & 604.81 & 508.838 & 508.840 & 508.840 \\
& 0.001 & & $10^{-4}$ & 0.002 & & $10^{-4}$ & 0.003 & & $10^{-4}$ \\
\hline \hline
\end{tabular}

\begin{tabular}{|c|ccc|}
\hline \hline Overall & MC & LB & MC (CV) \\
\cline { 2 - 4 } time $(\mathrm{sec})$ & 32.023 & 0.117 & 3.543 \\
\hline
\end{tabular}

Payer Swaptions (OTMF: $1.15 \times$ ATMF)

\begin{tabular}{|c|ccc|ccc|ccc|}
\hline Opt. Mat. & \multicolumn{5}{|c|}{1} & \multicolumn{3}{|c|}{2} & \multicolumn{3}{|c|}{5} \\
\hline Swap length & MC & LB & MC (CV) & MC & LB & MC (CV) & MC & LB & MC (CV) \\
\hline \multirow{2}{*}{1} & 1.571 & 1.570 & 1.570 & 2.823 & 2.824 & 2.824 & 3.798 & 3.794 & 3.794 \\
& 0.003 & & $10^{-4}$ & 0.005 & & $10^{-4}$ & 0.006 & & $10^{-4}$ \\
\hline \multirow{2}{*}{2} & 1.065 & \multirow{2}{*}{065} & 1.065 & 2.611 & 2.612 & 2.612 & 4.324 & 4.322 & 4.322 \\
& 0.003 & & $10^{-4}$ & 0.006 & & $10^{-4}$ & 0.008 & & $10^{-4}$ \\
\hline \multirow{2}{*}{5} & 0.150 & 0.150 & 0.150 & 0.904 & 0.905 & 0.905 & 2.569 & 2.569 & 2.57 \\
& 0.001 & & $10^{-4}$ & 0.004 & & $10^{-4}$ & 0.007 & & $10^{-4}$ \\
\hline \multirow{2}{*}{10} & 0.003 & 0.003 & 0.003 & 0.076 & 0.076 & 0.076 & 0.517 & 0.516 & 0.517 \\
& $10^{-4}$ & & $10^{-4}$ & 0.001 & & $10^{-4}$ & 0.003 & & $10^{-4}$ \\
\hline \hline
\end{tabular}

\begin{tabular}{|c|ccc|}
\hline Overall & MC & LB & MC (CV) \\
\cline { 2 - 4 } time $(\mathrm{sec})$ & 32.024 & 0.113 & 3.541 \\
\hline
\end{tabular}

Table 2: The three tables represent matrices of swaption prices at three different strikes for the 3factors Gaussian model. The bottom line of each table provides the overall computation time for the different pricing methods. For each swaption we report the price in basis points estimated with the Monte Carlo method, MC, the Lower Bound approximation (LB) and the Monte Carlo method with the control variable technique, $\mathrm{MC}(\mathrm{CV})$. Monte Carlo prices without and with control variable method are estimated using $10^{7}$ and respectively, $10^{5}$ simulations, antithetic variates method and the exact probability distribution of the state variables at the maturity date of the swaption. Below each Monte Carlo price, the confidence interval at $97.5 \%$ is reported in basis point. 


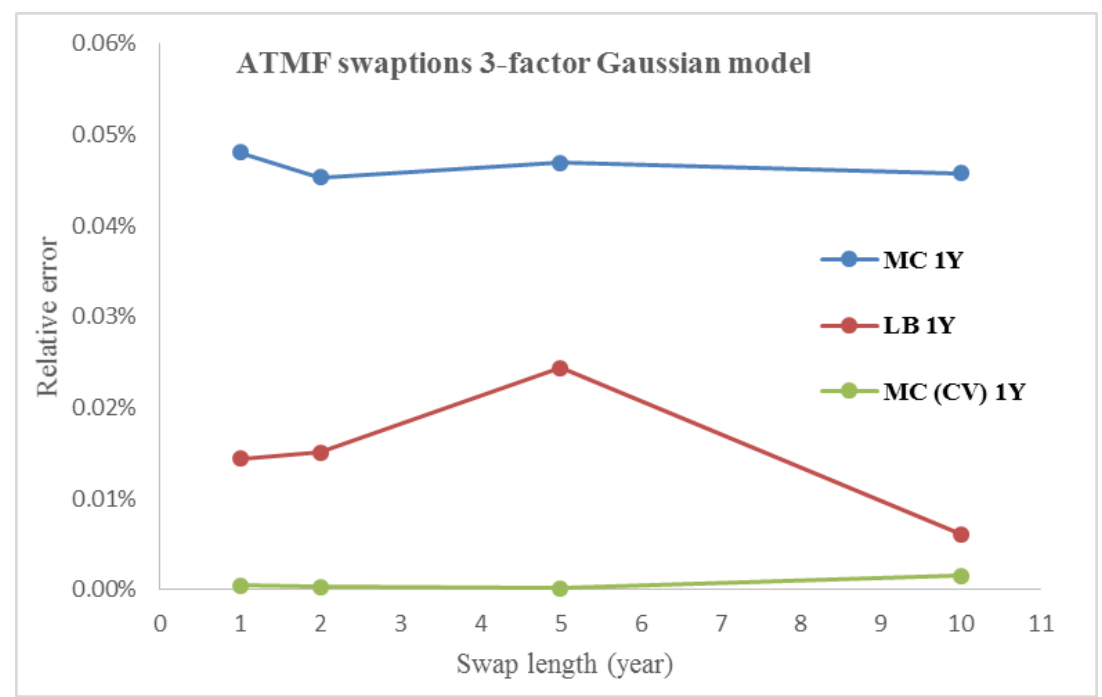

(a) ATMF-1 year maturity

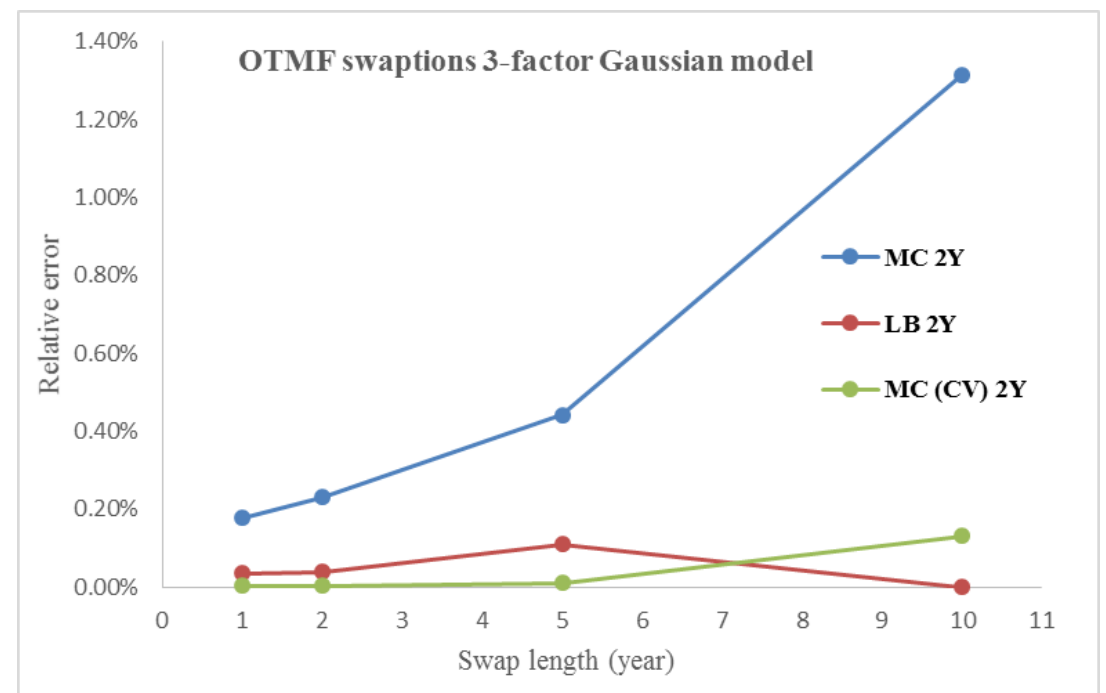

(b) OTMF-2 years maturity

Figure 1: Three-factor Gaussian model: relative error in percentage. The error of the lower bound is the difference from Monte Carlo value. 


\section{Two-factor Cox-Ingersoll-Ross model}

\begin{tabular}{|c|c|c|c|c|c|c|c|c|c|}
\hline \multicolumn{10}{|c|}{ Payer Swaptions (ATMF) } \\
\hline Opt. Mat. & \multicolumn{3}{|c|}{1} & \multicolumn{3}{|c|}{2} & \multicolumn{3}{|c|}{5} \\
\hline Swap length & $\mathrm{MC}$ & LB & $\mathrm{MC}(\mathrm{CV})$ & $\mathrm{MC}$ & $\mathrm{LB}$ & $\mathrm{MC}(\mathrm{CV})$ & $\mathrm{MC}$ & LB & $\mathrm{MC}(\mathrm{CV})$ \\
\hline \multirow{2}{*}{1} & 48.490 & 48.466 & 48.466 & 59.386 & 59.361 & 59.361 & 67.006 & 66.970 & 66.970 \\
\hline & 0.044 & & $10^{-4}$ & 0.054 & & $10^{-4}$ & 0.060 & & $10^{-4}$ \\
\hline \multirow{2}{*}{2} & 85.908 & 85.871 & 85.871 & 106.938 & 106.890 & 106.890 & 123.894 & 123.830 & 123.830 \\
\hline & 0.078 & & $10^{-4}$ & 0.098 & & $10^{-4}$ & 0.113 & & $10^{-4}$ \\
\hline \multirow{2}{*}{5} & 169.474 & 169.427 & 169.428 & 216.993 & 216.879 & 216.881 & 261.486 & 261.366 & 261.368 \\
\hline & 0.157 & & $10^{-4}$ & 0.202 & & $10^{-4}$ & 0.246 & & $10^{-4}$ \\
\hline \multirow{2}{*}{10} & 265.862 & 265.779 & 265.818 & 345.184 & 344.949 & 344.992 & 423.059 & 422.857 & 422.887 \\
\hline & 0.251 & & 0.004 & 0.331 & & 0.005 & 0.418 & & 0.004 \\
\hline \multirow{2}{*}{$\begin{array}{c}\text { Overall } \\
\text { time }(\mathrm{sec})\end{array}$} & $\mathrm{MC}$ & $\mathrm{LB}$ & $\mathrm{MC}(\mathrm{CV})$ & & & & & & \\
\hline & 23.118 & 1.456 & 1.924 & & & & & & \\
\hline
\end{tabular}

Payer Swaptions (ITMF: $0.85 \times$ ATMF)

\begin{tabular}{|c|ccc|ccc|ccc|}
\hline Opt. Mat. & \multicolumn{3}{|c|}{1} & \multicolumn{3}{c|}{2} & \multicolumn{3}{|c|}{5} \\
\hline Swap length & $\mathrm{MC}$ & $\mathrm{LB}$ & $\mathrm{MC}(\mathrm{CV})$ & $\mathrm{MC}$ & $\mathrm{LB}$ & $\mathrm{MC}(\mathrm{CV})$ & $\mathrm{MC}$ & $\mathrm{LB}$ & $\mathrm{MC}(\mathrm{CV})$ \\
\hline \multirow{2}{*}{1} & 107.584 & 107.577 & 107.577 & 116.846 & 116.839 & 116.839 & 114.947 & 114.930 & 114.930 \\
& 0.025 & & $10^{-4}$ & 0.034 & & $10^{-4}$ & 0.043 & $10^{-4}$ \\
\hline \multirow{2}{*}{2} & 208.047 & 208.037 & 208.037 & 222.373 & 222.363 & 222.363 & 217.236 & 217.208 & 217.208 \\
& 0.040 & & $10^{-4}$ & 0.058 & & $10^{-4}$ & 0.078 & $10^{-4}$ \\
\hline \multirow{2}{*}{5} & 475.686 & 475.668 & 475.669 & 493.334 & 493.301 & 493.301 & 473.362 & 473.330 & 473.331 \\
& 0.065 & & $10^{-4}$ & 0.109 & & $10^{-4}$ & 0.165 & $10^{-4}$ \\
\hline \multirow{2}{*}{10} & 812.501 & 812.470 & 812.482 & 825.291 & 825.202 & 825.219 & 778.599 & 778.559 & 778.573 \\
& 0.092 & & 0.002 & 0.168 & & 0.003 & 0.275 & & 0.002 \\
\hline
\end{tabular}

\begin{tabular}{c|ccc|} 
Overall & MC & LB & MC (CV) \\
\cline { 2 - 4 } time $(\mathrm{sec})$ & 23.121 & 1.199 & 1.667 \\
\hline
\end{tabular}

Payer Swaptions (OTMF: $1.15 \times$ ATMF)

\begin{tabular}{|c|ccc|ccc|ccc|}
\hline Opt. Mat. & \multicolumn{3}{|c|}{1} & \multicolumn{3}{|c|}{2} & \multicolumn{3}{|c|}{5} \\
\hline Swap length & MC & LB & MC (CV) & MC & LB & MC (CV) & MC & LB & MC (CV) \\
\hline \multirow{2}{*}{1} & 16.004 & 15.973 & 15.973 & 24.476 & 24.446 & 24.446 & 34.601 & 34.546 & 34.546 \\
& 0.062 & & $10^{-4}$ & 0.072 & & $10^{-4}$ & 0.077 & $10^{-4}$ \\
\hline \multirow{2}{*}{2} & 23.777 & 23.724 & 23.724 & 40.022 & 39.964 & 39.964 & 61.943 & 61.838 & 61.838 \\
& 0.113 & & $10^{-4}$ & 0.134 & & $10^{-4}$ & 0.146 & $10^{-4}$ \\
\hline \multirow{2}{*}{5} & 33.668 & 33.565 & 33.567 & 68.868 & 68.740 & 68.742 & 124.627 & 124.396 & 124.399 \\
& 0.239 & & $10^{-4}$ & 0.288 & & 0.001 & 0.323 & 0.001 \\
\hline \multirow{2}{*}{10} & 42.602 & 42.425 & 42.459 & 99.260 & 99.004 & 99.045 & 196.625 & 196.230 & 196.266 \\
& 0.394 & & 0.005 & 0.483 & & 0.005 & 0.555 & 0.005 \\
\hline \hline
\end{tabular}

\begin{tabular}{c|ccc|} 
Overall & MC & LB & MC (CV) \\
\cline { 2 - 4 } time $(\mathrm{sec})$ & 23.121 & 1.314 & 1.782 \\
\hline
\end{tabular}

Table 3: The three tables represent matrices of swaption prices at three different strikes for the 2-factor C.I.R. model. The bottom line of each table provides the overall computation time for the different pricing methods. For each swaption we report the price in basis points estimated with the Monte Carlo method, MC, the Lower Bound approximation (LB) and the Monte Carlo method with the control variable technique, $\mathrm{MC}(\mathrm{CV})$. Monte Carlo prices without and with control variable method are estimated using $10^{7}$ and respectively, $10^{5}$ simulations and the exact probability distribution of the state variables at the maturity date of the swaption. Below each Monte Carlo price, the confidence interval at $97.5 \%$ is reported in basis point. 


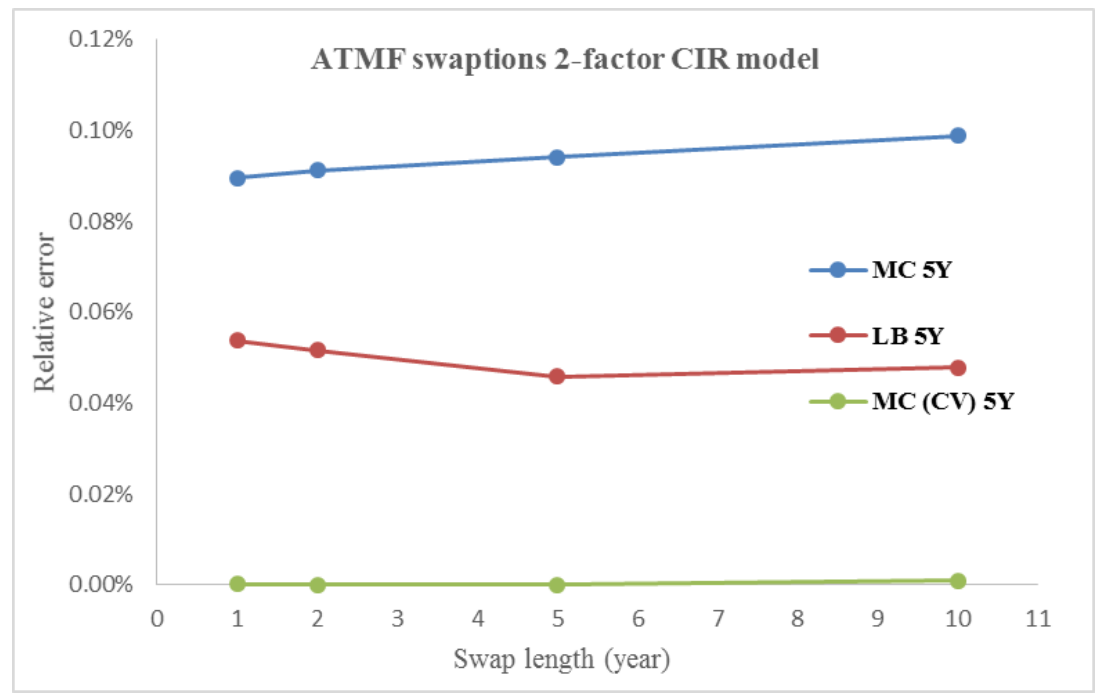

(a) ATMF-5 year maturity

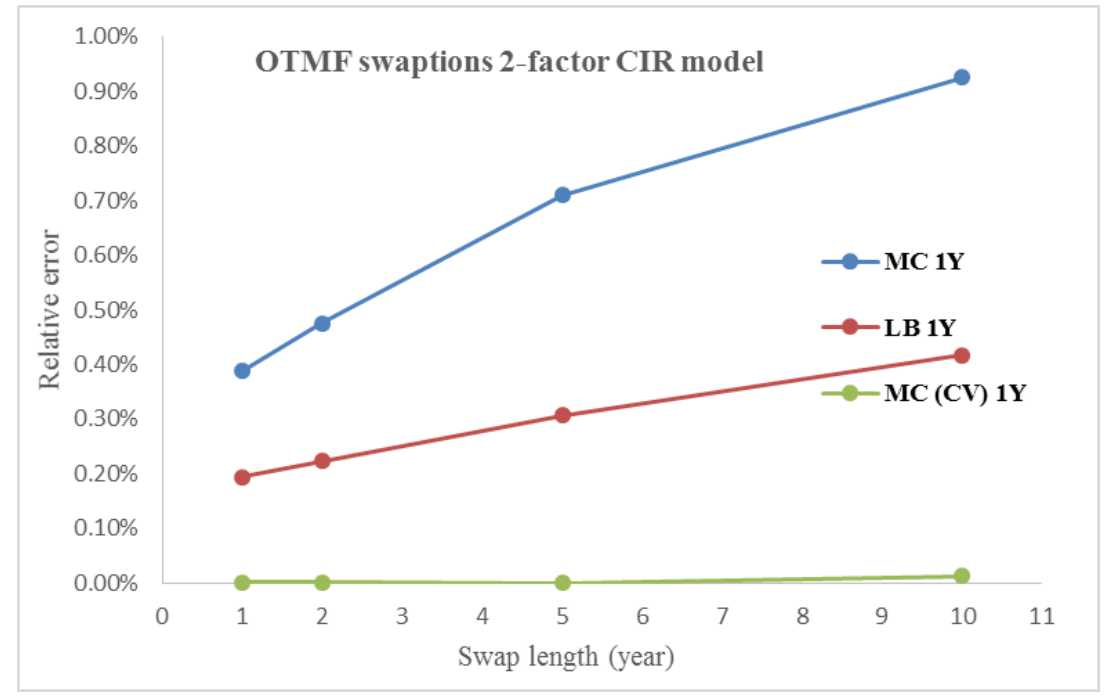

(b) OTMF-1 years maturity

Figure 2: Two-factor Cox-Ingersoll-Ross model: relative error in percentage. The error of the lower bound is the difference from Monte Carlo value. 


\section{Two-factor Gaussian model with Double Exponential Jumps}

\begin{tabular}{|c|c|c|c|c|c|c|c|c|c|}
\hline \multicolumn{10}{|c|}{ Payer Swaptions (ATMF) } \\
\hline Opt. Mat. & \multicolumn{3}{|c|}{1} & \multicolumn{3}{|c|}{2} & \multicolumn{3}{|c|}{5} \\
\hline Swap length & $\mathrm{MC}$ & $\mathrm{LB}$ & $\mathrm{MC}(\mathrm{CV})$ & $\mathrm{MC}$ & $\mathrm{LB}$ & $\mathrm{MC}(\mathrm{CV})$ & $\mathrm{MC}$ & LB & $\mathrm{MC}(\mathrm{CV})$ \\
\hline \multirow{2}{*}{1} & 44.013 & 44.046 & 44.046 & 52.773 & 52.766 & 52.766 & 59.145 & 59.204 & 59.204 \\
\hline & 0.063 & & $10^{-4}$ & 0.074 & & $10^{-4}$ & 0.082 & & $10^{-4}$ \\
\hline \multirow{2}{*}{2} & 82.968 & 83.042 & 83.042 & 102.255 & 102.242 & 102.242 & 119.461 & 119.629 & 119.629 \\
\hline & 0.117 & & $10^{-4}$ & 0.143 & & $10^{-4}$ & 0.165 & & $10^{-4}$ \\
\hline \multirow{2}{*}{5} & 96.950 & 97.030 & 97.031 & 120.664 & 120.652 & 120.652 & 142.807 & 143.021 & 143.021 \\
\hline & 0.137 & & $10^{-4}$ & 0.169 & & $10^{-4}$ & 0.196 & & $10^{-4}$ \\
\hline \multirow{2}{*}{10} & 97.542 & 97.623 & 97.623 & 121.396 & 121.384 & 121.384 & 143.643 & 143.858 & 143.858 \\
\hline & 0.138 & & $10^{-4}$ & 0.170 & & $10^{-4}$ & 0.198 & & $10^{-4}$ \\
\hline \multirow{2}{*}{$\begin{array}{c}\text { Overall } \\
\text { time (sec) }\end{array}$} & $\mathrm{MC}$ & LB & $\mathrm{MC}(\mathrm{CV})$ & & & & & & \\
\hline & $5.396 \times 10^{3}$ & 2.643 & 84.485 & & & & & & \\
\hline
\end{tabular}

Payer Swaptions (ITMF: $0.85 \times$ ATMF)

\begin{tabular}{|c|ccc|ccc|ccc|}
\hline Opt. Mat. & \multicolumn{3}{|c|}{1} & \multicolumn{3}{c|}{2} & \multicolumn{3}{c|}{5} \\
\hline Swap length & $\mathrm{MC}$ & $\mathrm{LB}$ & $\mathrm{MC}(\mathrm{CV})$ & $\mathrm{MC}$ & $\mathrm{LB}$ & $\mathrm{MC}(\mathrm{CV})$ & $\mathrm{MC}$ & $\mathrm{LB}$ & $\mathrm{MC}(\mathrm{CV})$ \\
\hline \multirow{2}{*}{1} & 63.821 & 63.878 & 63.878 & 68.253 & 68.267 & 68.267 & 69.103 & 69.164 & 69.164 \\
& 0.074 & & $10^{-4}$ & 0.084 & & $10^{-4}$ & 0.088 & $10^{-4}$ \\
\hline \multirow{2}{*}{2} & 138.588 & 138.729 & 138.729 & 148.623 & 148.667 & 148.667 & 153.982 & 154.172 & 154.172 \\
& 0.146 & & $10^{-4}$ & 0.169 & & $10^{-4}$ & 0.184 & $10^{-4}$ \\
\hline \multirow{2}{*}{5} & 349.841 & 350.150 & 350.150 & 347.712 & 347.888 & 347.888 & 340.988 & 341.320 & 341.320 \\
& 0.214 & & $10^{-4}$ & 0.250 & & $10^{-4}$ & 0.275 & $10^{-4}$ \\
\hline \multirow{2}{*}{10} & 904.579 & 904.933 & 904.934 & 887.761 & 887.995 & 887.995 & 856.180 & 856.559 & 856.559 \\
& 0.215 & & $10^{-4}$ & 0.264 & & $10^{-4}$ & 0.305 & & $10^{-4}$ \\
\hline \hline
\end{tabular}

\begin{tabular}{c|ccc|} 
Overall & MC & LB & MC (CV) \\
\cline { 2 - 3 } time $(\mathrm{sec})$ & $5.396 \times 10^{3}$ & 2.643 & 84.057 \\
\end{tabular}

Payer Swaptions (OTMF: $1.15 \times$ ATMF)

\begin{tabular}{|c|ccc|ccc|ccc|}
\hline Opt. Mat. & \multicolumn{3}{|c|}{1} & \multicolumn{3}{|c|}{2} & \multicolumn{3}{c|}{5} \\
\hline Swap length & MC & LB & MC (CV) & MC & LB & MC (CV) & MC & LB & MC (CV) \\
\hline \multirow{2}{*}{1} & 28.669 & 28.679 & 28.679 & 39.757 & 39.729 & 39.729 & 50.158 & 50.215 & 50.215 \\
& 0.051 & & $10^{-4}$ & 0.065 & & $10^{-4}$ & 0.076 & $10^{-4}$ \\
\hline \multirow{2}{*}{2} & 44.305 & 44.319 & 44.319 & 66.449 & 66.396 & 66.396 & 90.386 & 90.535 & 90.535 \\
& 0.087 & & $10^{-4}$ & 0.117 & & $10^{-4}$ & 0.144 & $10^{-4}$ \\
\hline \multirow{2}{*}{5} & 9.461 & 9.449 & 9.449 & 22.651 & 22.573 & 22.573 & 41.176 & 41.275 & 41.275 \\
& 0.041 & & $10^{-4}$ & 0.072 & & $10^{-4}$ & 0.105 & $10^{-4}$ \\
\hline \multirow{2}{*}{10} & 0.051 & 0.048 & 0.048 & 0.299 & 0.304 & 0.304 & 1.563 & 1.564 & 1.564 \\
& 0.005 & & $10^{-4}$ & 0.009 & & $10^{-4}$ & 0.019 & & $10^{-4}$ \\
\hline \hline
\end{tabular}

\begin{tabular}{|c|ccc|}
\hline \hline Overall & MC & LB & MC (CV) \\
\cline { 2 - 4 } time $(\mathrm{sec})$ & $5.396 \times 10^{3}$ & 2.643 & 84.070 \\
\hline
\end{tabular}

Table 4: The three tables represent matrices of swaption prices at three different strikes for the 2factor Gaussian model with Double Exponential Jumps. The bottom line of each table provides the overall computation time for the different pricing methods. For each swaption we report the price in basis points estimated with the Monte Carlo method, MC, the Lower Bound approximation (LB) and the Monte Carlo method with the control variable technique, $\mathrm{MC}(\mathrm{CV})$. Monte Carlo (MC) price is estimated using 4 millions simulations, an Euler scheme with a time step equal to 0.0025 and the antithetic variates technique. Monte Carlo is also performed using $10^{5}$ simulations and control variates method. Below each Monte Carlo price, the confidence interval at $97.5 \%$ is reported in basis point. 


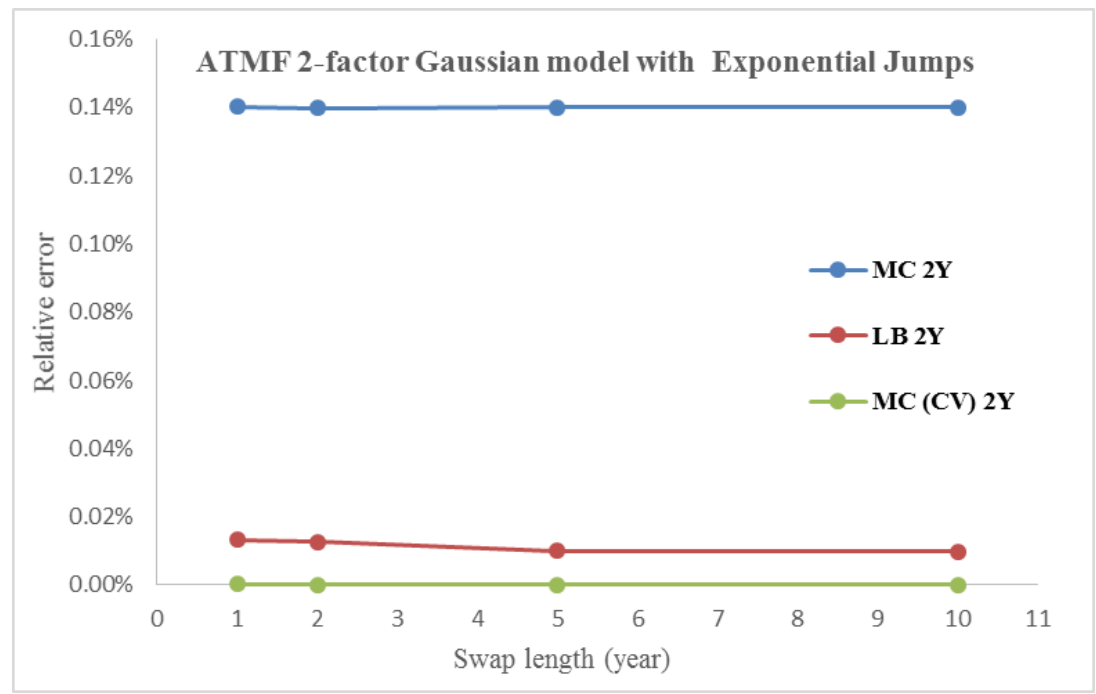

(a) ATMF-2 year maturity

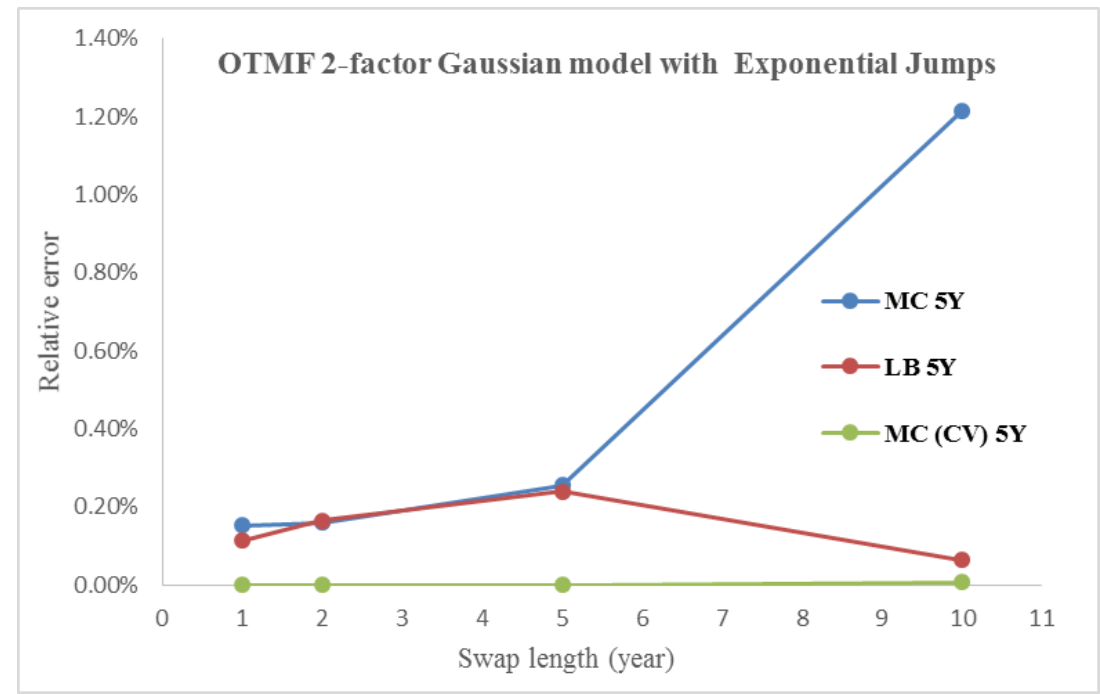

(b) OTMF-5 years maturity

Figure 3: Two-factor Gaussian model with Double Exponential Jumps: relative error in percentage. The error of the lower bound is the difference from Monte Carlo value. 


\section{Balduzzi, Das, Foresi and Sundaram model}

\begin{tabular}{|c|c|c|c|c|c|c|c|c|c|}
\hline \multicolumn{10}{|c|}{ Payer Swaptions (ATMF) } \\
\hline Opt. Mat. & \multicolumn{3}{|c|}{1} & \multicolumn{3}{|c|}{2} & \multicolumn{3}{|c|}{5} \\
\hline Swap length & $\mathrm{MC}$ & LB & $\mathrm{MC}(\mathrm{CV})$ & $\mathrm{MC}$ & LB & $\mathrm{MC}(\mathrm{CV})$ & $\mathrm{MC}$ & $\mathrm{LB}$ & $\mathrm{MC}(\mathrm{CV})$ \\
\hline \multirow{2}{*}{1} & 85.08 & 85.00 & 85.00 & 102.24 & 102.17 & 102.16 & 97.45 & 97.45 & 97.45 \\
\hline & 0.06 & & $10^{-3}$ & 0.07 & & $10^{-3}$ & 0.06 & & $10^{-3}$ \\
\hline \multirow{2}{*}{2} & 148.39 & 148.27 & 148.26 & 177.40 & 177.28 & 177.27 & 167.37 & 167.38 & 167.38 \\
\hline & 0.10 & & $10^{-3}$ & 0.11 & & $10^{-3}$ & 0.09 & & $10^{-3}$ \\
\hline \multirow{2}{*}{5} & 250.22 & 250.01 & 250.01 & 296.89 & 296.68 & 296.68 & 276.29 & 276.28 & 276.28 \\
\hline & 0.17 & & $10^{-3}$ & 0.18 & & $10^{-3}$ & 0.15 & & $10^{-3}$ \\
\hline \multirow{2}{*}{10} & 290.96 & 290.71 & 290.72 & 345.34 & 345.10 & 345.10 & 321.69 & 321.68 & 321.68 \\
\hline & 0.19 & & $10^{-3}$ & 0.21 & & $10^{-3}$ & 0.17 & & $10^{-3}$ \\
\hline \multirow{2}{*}{$\begin{array}{c}\text { Overall } \\
\text { time }(\mathrm{sec})\end{array}$} & $\mathrm{MC}$ & LB & $\mathrm{MC}(\mathrm{CV})$ & & & & & & \\
\hline & $12.256 \times 10^{3}$ & 894 & $1.139 \times 10^{3}$ & & & & & & \\
\hline
\end{tabular}

Payer Swaptions (ITMF: $0.85 \times$ ATMF)

\begin{tabular}{|c|ccc|ccc|ccc|}
\hline Opt. Mat. & \multicolumn{4}{|c|}{1} & \multicolumn{3}{|c|}{2} & \multicolumn{3}{|c|}{5} \\
\hline Swap length & MC & LB & MC (CV) & MC & LB & MC (CV) & MC & LB & MC (CV) \\
\hline \multirow{2}{*}{1} & 122.53 & 122.44 & 122.44 & 148.64 & 148.57 & 148.57 & 152.26 & 152.25 & 152.25 \\
& 0.05 & & $10^{-3}$ & 0.06 & & $10^{-3}$ & 0.04 & $10^{-3}$ \\
\hline \multirow{2}{*}{2} & 234.64 & 234.51 & 234.50 & 277.64 & 277.52 & 277.51 & 277.32 & 277.31 & 277.31 \\
& 0.09 & & $10^{-3}$ & 0.1 & & $10^{-3}$ & 0.07 & $10^{-3}$ \\
\hline \multirow{2}{*}{5} & 521.94 & 521.72 & 521.72 & 578.32 & 578.11 & 578.11 & 543.71 & 543.68 & 543.68 \\
& 0.11 & & $10^{-3}$ & 0.12 & & $10^{-3}$ & 0.08 & 0 & 0 \\
\hline \multirow{2}{*}{10} & 881.46 & 881.21 & 881.21 & 908.49 & 908.26 & 908.26 & 799.72 & 799.70 & 799.70 \\
& 0.07 & & $10^{-3}$ & 0.08 & & $10^{-3}$ & 0.07 & & $10^{-3}$ \\
\hline
\end{tabular}

\begin{tabular}{|c|ccc|}
\hline \hline $\begin{array}{c}\text { Overall } \\
\text { time }(\mathrm{sec})\end{array}$ & $\mathrm{MC}$ & $\mathrm{LB}$ & $\mathrm{MC}(\mathrm{CV})$ \\
\cline { 2 - 4 } & $12.256 \times 10^{3}$ & 930 & $1.176 \times 10^{3}$ \\
\hline
\end{tabular}

Payer Swaptions (OTMF: $1.15 \times$ ATMF)

\begin{tabular}{|c|ccc|ccc|ccc|}
\hline Opt. Mat. & \multicolumn{3}{|c|}{1} & \multicolumn{3}{|c|}{2} & \multicolumn{3}{c|}{5} \\
\hline Swap length & MC & LB & MC (CV) & MC & LB & MC (CV) & MC & LB & MC (CV) \\
\hline \multirow{2}{*}{1} & 55.91 & 55.84 & 55.84 & 66.35 & 66.29 & 66.28 & 57.30 & 57.30 & 57.30 \\
& 0.06 & & $10^{-3}$ & 0.07 & & $10^{-3}$ & 0.06 & $10^{-3}$ \\
\hline \multirow{2}{*}{2} & 85.74 & 85.65 & 85.64 & 104.05 & 103.95 & 103.94 & 90.40 & 90.40 & 90.40 \\
& 0.09 & & $10^{-3}$ & 0.11 & & $10^{-3}$ & 0.09 & $10^{-3}$ \\
\hline \multirow{2}{*}{5} & 92.5 & 92.38 & 92.38 & 124.12 & 123.99 & 123.98 & 113.7 & 113.69 & 113.69 \\
& 0.13 & & $10^{-3}$ & 0.16 & & $10^{-3}$ & 0.13 & $10^{-3}$ \\
\hline \multirow{2}{*}{10} & 48.09 & 48.00 & 48.01 & 81.30 & 81.18 & 81.17 & 86.16 & 86.15 & 86.15 \\
& 0.10 & & $10^{-3}$ & 0.14 & & $10^{-3}$ & 0.13 & & $10^{-3}$ \\
\hline \hline
\end{tabular}

\begin{tabular}{|c|ccc|}
\hline Overall & $\mathrm{MC}$ & $\mathrm{LB}$ & $\mathrm{MC}(\mathrm{CV})$ \\
\cline { 2 - 4 } time $(\mathrm{sec})$ & $12.256 \times 10^{3}$ & 928 & $1.172 \times 10^{3}$ \\
\hline
\end{tabular}

Table 5: The three tables represent matrices of swaption prices at three different strikes for the BDFS. The bottom line of each table provides the overall computation time for the different pricing methods. For each swaption we report the price in basis points estimated with the Monte Carlo method, MC, the Lower Bound approximation (LB) and the Monte Carlo method with the control variable technique, MC (CV). Monte Carlo (MC) price is estimated using 4 millions simulations, an Euler scheme with a time step equal to 0.0005 and the antithetic variates technique. Monte Carlo is also performed using $10^{5}$ simulations and control variates method. Below each Monte Carlo price, the confidence interval at $97.5 \%$ is reported in basis point. 


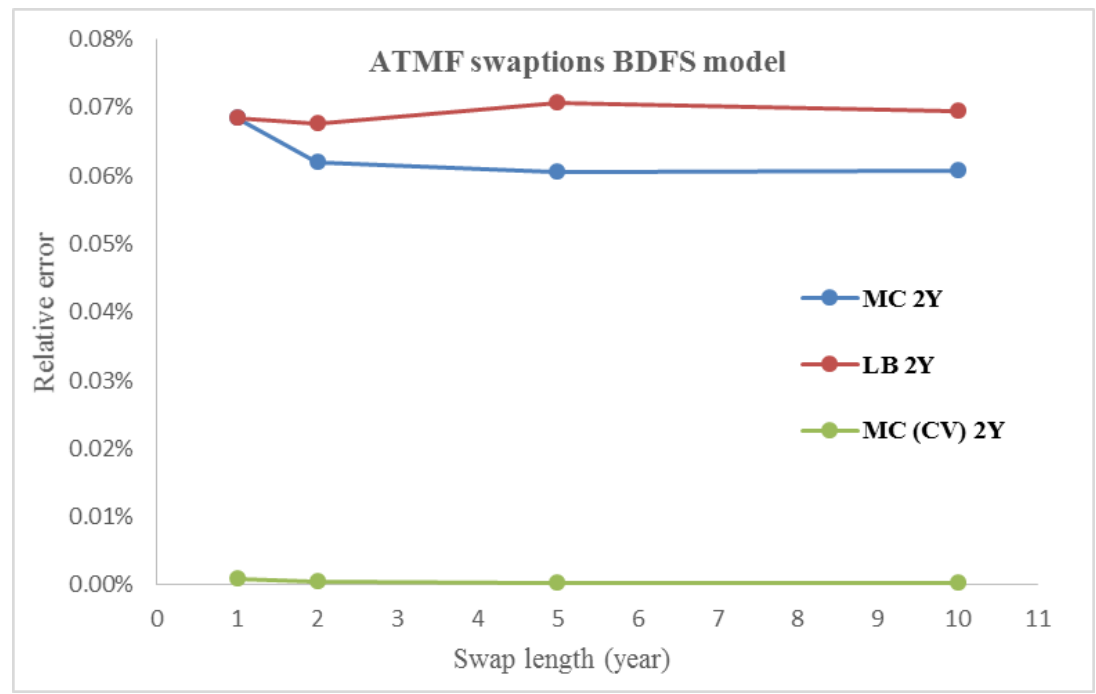

(a) ATMF-2 year maturity

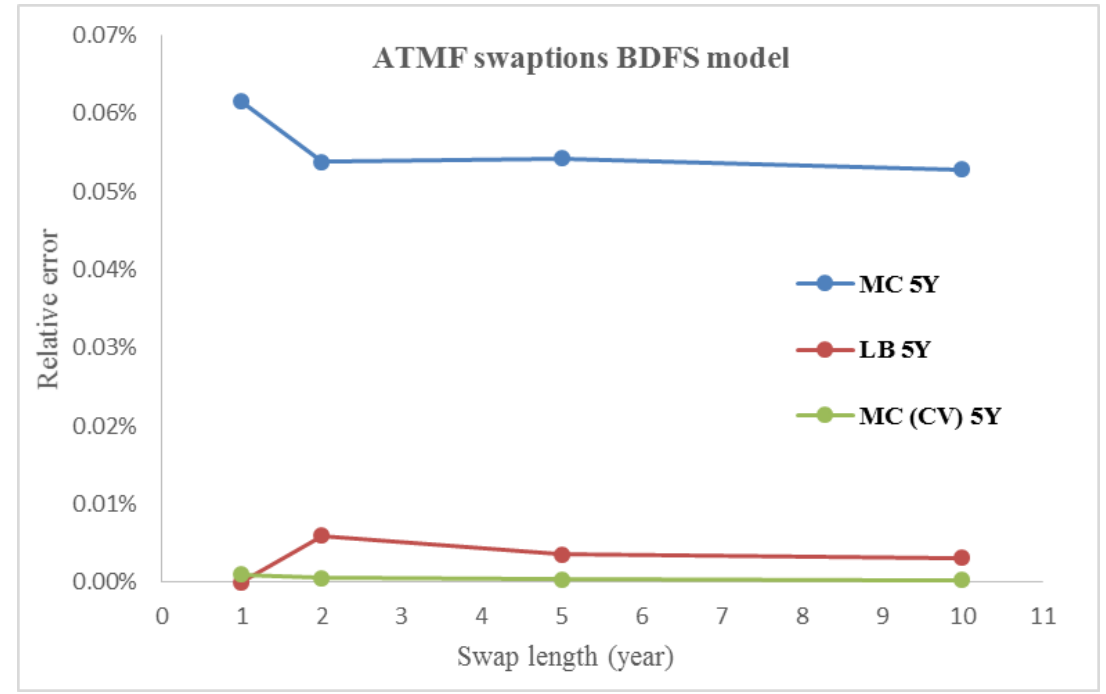

(b) ATMF-5 years maturity

Figure 4: Balduzzi, Das, Foresi and Sundaram model: relative error in percentage. The error of the lower bound is the difference from Monte Carlo value. 


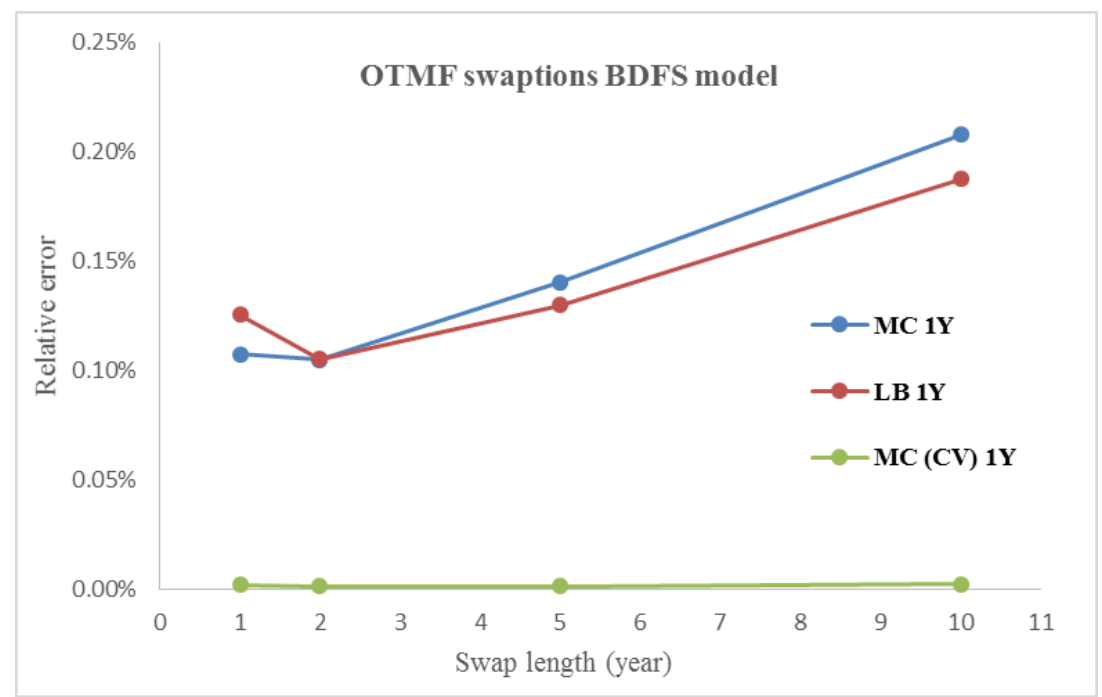

(a) OTMF-2 year maturity

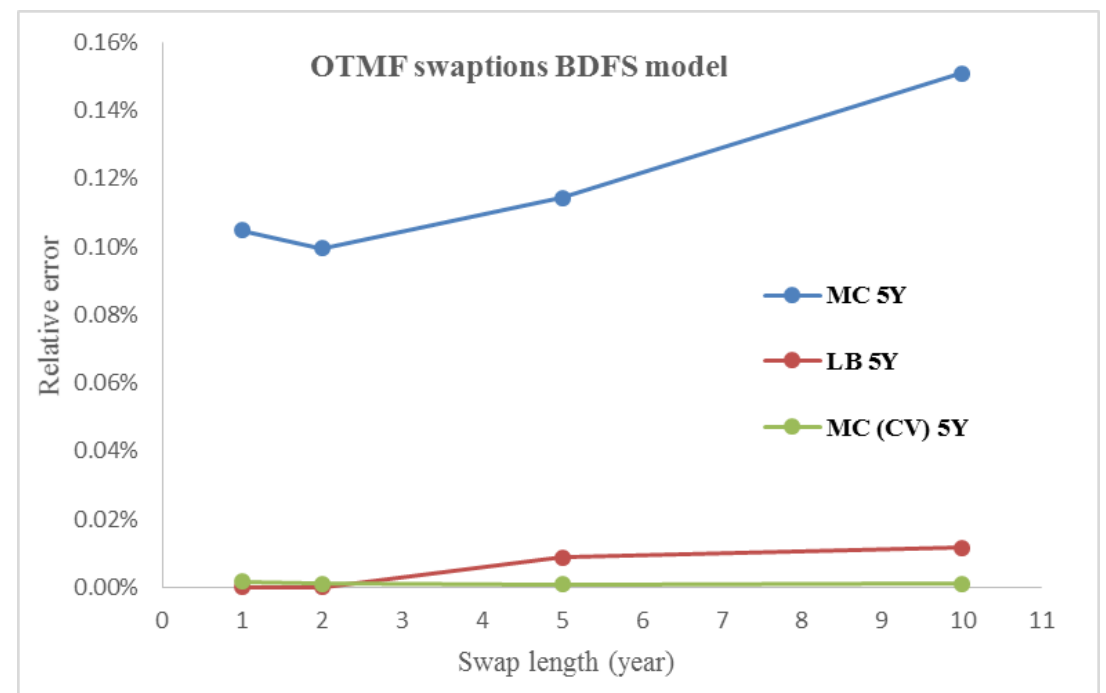

(b) OTMF-5 years maturity

Figure 5: Balduzzi, Das, Foresi and Sundaram model: relative error in percentage. The error of the lower bound is the difference from Monte Carlo value. 


\section{References}

P. Balduzzi, S.R. Das, S. Foresi and R. Sundaram. A simple approach to three-factor affine term structure models. The Journal of Fixed Income,1996.

R. Caldana, G. Fusai, A. Gnoatto and M. Grasselli. General closed-form basket option pricing bounds. Working paper, posted at ssrn.com in January 2014.

P. Carr and D.P. Madan. Option valuation using the Fast Fourier Transform. Journal of Computational Finance, 2:61-73, 2000.

P. Collin-Dufresne and R.S. Goldstein. Pricing Swaptions Within an Affine Framework. The Journal of Derivatives, 2002.

D. Duffie, P. Pan and K. Singleton. Transform Analysis and Asset Pricing for Affine Jump-Diffusions. Econometrica, 68:1343-1376, 2000.

D. Duffie and K. Singleton. An Econometric Model of the Term Structure of Interest-Rate Swap Yields. J. Finance, 52:1287-1321, 1997.

D. Duffie and . A yield-factor model of interest rates. Mathematical Finance, 6:379-406, 1996.

F. Jamshidian. An exact bond option pricing formula. Journal of Finance, 44: 205-209, 1989.

C. Munk. Stochastic Duration and Fast Coupon Bond Option Pricing in Multi-Factor Models. Rev. Derivat 3:157-181, 1999.

D.F.. Schrager and A.J. Pelsser. Pricing Swaptions and Coupon Bond Options in Affine Term Structure Models. Mathematical Finance, 16:673-694, 2006.

K.J. Singleton and L. Umantsev. Pricing Coupon-Bond Options and Swaptions in Affine Term Structure Models. Mathematical Finance, 12:427-446, 2002.

\section{A Appendix}

\section{A.1 Proof Proposition 1}

We consider the lower bound to the swaption price as in formula (2.2) for affine models:

$$
L B\left(k ; t, T,\left\{T_{h}\right\}_{h=1}^{n}, R\right)=P(t, T) \cdot \mathbb{E}_{t}^{T}\left[\left(\sum_{h=1}^{n} w_{h} e^{\mathbf{b}_{h}^{\top} \mathbf{X}(T)+a_{h}}-1\right) I(\mathcal{G})\right]^{+}
$$

where the set $\mathcal{G}=\{\omega \in \Omega: g(\mathbf{X}(T)) \geq k\}=\left\{\omega \in \Omega: \boldsymbol{\beta}^{\top} \mathbf{X}(T)+\alpha \geq k\right\}$.

According to Carr and Madan (2000), we introduce the dampening factor $e^{\delta k}$, then we apply the Fourier Transform with respect to the variable $k$ to the T-forward expected value and we obtain:

$$
\begin{aligned}
\psi_{\delta}(\gamma) & =\int_{-\infty}^{+\infty} e^{i \gamma k+\delta k} \mathbb{E}_{t}^{T}\left[\left(\sum_{h=1}^{n} w_{h} e^{\mathbf{b}_{h}^{\top} \mathbf{X}(T)+a_{h}}-1\right) I(g(\mathbf{X}(T)) \geq k)\right] d k= \\
& =\mathbb{E}_{t}^{T}\left[\left(\sum_{h=1}^{n} w_{h} e^{\mathbf{b}_{h}^{\top} \mathbf{X}(T)+a_{h}}-1\right) \int_{-\infty}^{+\infty} e^{i \gamma k+\delta k} I\left(\boldsymbol{\beta}^{\top} \mathbf{X}(T)+\alpha \geq k\right) d k\right]= \\
& =\mathbb{E}_{t}^{T}\left[\left(\sum_{h=1}^{n} w_{h} e^{\mathbf{b}_{h}^{\top} \mathbf{X}(T)+a_{h}}-1\right) \int_{-\infty}^{\boldsymbol{\beta}^{\top} \mathbf{X}(T)+\alpha} e^{i \gamma k+\delta k} d k\right]
\end{aligned}
$$

Since the dampening factor $\delta$ is positive, then the module of the integrand function decays exponentially for $k \rightarrow-\infty$ and the Fourier Transform is well defined, so:

$$
\psi_{\delta}(\gamma)=\mathbb{E}_{t}^{T}\left[\left(\sum_{h=1}^{n} w_{h} e^{\mathbf{b}_{h}^{\top} \mathbf{X}(T)+a_{h}}-1\right) e^{(i \gamma+\delta)\left(\boldsymbol{\beta}^{\top} \mathbf{X}(T)\right)}\right] \frac{e^{(i \gamma+\delta) \alpha}}{i \gamma+\delta}
$$


Using the characteristic function of $\mathbf{X}$, calculated under the T-forward measure: $\Phi(\boldsymbol{\lambda})=\mathbb{E}_{t}^{T}\left[e^{i \boldsymbol{\lambda}^{\top} \mathbf{X}}\right]$, the function $\psi_{\delta}(\gamma)$ can be written as:

$$
\psi_{\delta}(\gamma)=\left(\sum_{h=1}^{n} w_{h} e^{a_{h}} \Phi\left(-i \mathbf{b}_{h}+(\gamma-i \delta) \boldsymbol{\beta}\right)-\Phi((\gamma-i \delta) \boldsymbol{\beta})\right) \frac{e^{(i \gamma+\delta) \alpha}}{i \gamma+\delta}
$$

Finally the lower bound is the maximum with respect to $k$ of the inverse transform of $\psi_{\delta}(\gamma)$ :

$$
L B\left(k ; t, T,\left\{T_{h}\right\}_{h=1}^{n}, R\right)=P(t, T) \frac{e^{-\delta k}}{\pi} \int_{0}^{+\infty} e^{-i \gamma k} \psi_{\delta}(\gamma) d \gamma
$$

\section{A.2 Proof of the analytical lower bound for Gaussian affine models}

$\mathbf{X} \sim N(\boldsymbol{\mu}, V)$ in T-forward measure and $g(\mathbf{X}(T))=\boldsymbol{\beta}^{\top} \mathbf{X}+\alpha \sim N\left(\boldsymbol{\beta}^{\top} \boldsymbol{\mu}+\alpha, \boldsymbol{\beta}^{\top} V \boldsymbol{\beta}\right)$

Then the approximate exercise region $\mathcal{G}$ becomes:

$$
\mathcal{G}=\{\omega \in \Omega: g(\mathbf{X}(T))>k\}=\{\omega \in \Omega: z>d\}
$$

where $z$ is a standard normal random variable and

$$
d=\frac{k-\boldsymbol{\beta}^{\top} \boldsymbol{\mu}-\alpha}{\sqrt{\boldsymbol{\beta}^{\top} V \boldsymbol{\beta}}} .
$$

The lower bound expression can be written using the law of iterative expectation:

$$
L B\left(k ; t, T,\left\{T_{h}\right\}_{h=1}^{n}, R\right)=P(t, T) \mathbb{E}_{t}^{T}\left[\mathbb{E}_{t}^{T}\left[\left(\sum_{h=1}^{n} w_{h} e^{\mathbf{b}_{h}{ }^{\top} \mathbf{X}(T)+a_{h}}-1\right) \mid z\right] I(z>d)\right]
$$

Conditionally to the random variable $z$, the variable $\mathbf{X}$ is distributed as a multivariate normal with mean and variance:

$$
\begin{aligned}
& \mathbb{E}_{t}^{T}[\mathbf{X} \mid z]=\boldsymbol{\mu}+z \cdot \mathbf{v} \\
& \operatorname{Var}(\mathbf{X} \mid z)=V-\mathbf{v} \mathbf{v}^{\top} \\
& \mathbf{v}=\frac{V \boldsymbol{\beta}}{\sqrt{\boldsymbol{\beta}^{\top} V \boldsymbol{\beta}}}
\end{aligned}
$$

We can now compute the inner expectation, using the normal distribution property:

$$
L B\left(k ; t, T,\left\{T_{h}\right\}_{h=1}^{n}, R\right)=P(t, T)\left(\sum_{h=1}^{n} w_{h} \mathbb{E}_{t}^{T}\left[e^{\mathbf{b}_{h}^{\top} \boldsymbol{\mu}+z \mathbf{b}_{h}^{\top} \mathbf{v}+\frac{1}{2} V_{h}} I(z>d)\right]-\mathbb{E}_{t}^{T}[I(z>d)]\right)
$$

where $V_{h}=\mathbf{b}_{h}^{\top}\left(V-\mathbf{v} \mathbf{v}^{\top}\right) \mathbf{b}_{h}$.

Maximizing with respect to $k$, involved in the definition of $\mathrm{d}$, we found the lower bound:

$$
L B\left(t, T,\left\{T_{h}\right\}_{h=1}^{n}, R\right)=\max _{k \in \mathbb{R}}\left(\sum_{h=1}^{n} w_{h} e^{a_{h}+\mathbf{b}_{h}^{\top} \boldsymbol{\mu}+\frac{1}{2} V_{h}+\frac{1}{2} d_{h}^{2}} N\left(d_{h}-d\right)-N(-d)\right)
$$

where $d_{h}=\mathbf{b}_{h}^{\top} \mathbf{v}$ and $N(x)$ is the cumulative distribution function of standard normal variable.

To make faster the optimization of the lower bound with respect to the parameter $k$, we compute the first order approximation of maximum point as a starting point. Equation for stationary points:

$$
\frac{\partial L B(t, k)}{\partial k}=\frac{P(t, T)}{\sqrt{\boldsymbol{\beta}^{\top} V \boldsymbol{\beta}}}\left(\sum_{h=1}^{n} w_{h} e^{a_{h}+\mathbf{b}_{h}^{\top} \boldsymbol{\mu}+\frac{1}{2} V_{h}+d_{h}^{2} / 2} N^{\prime}\left(-\left(d-d_{h}\right)\right)-N^{\prime}(-d)\right)=0
$$

Taylor first order expansion:

$$
N^{\prime}\left(-\left(d-d_{h}\right)\right)=N^{\prime}(-d)+d_{h} N^{\prime \prime}(-d)+o\left(d_{h}\right)=N^{\prime}(-d)\left(1+d d_{h}\right)+o\left(d_{h}\right)
$$


We substitute the first order expansion in the derivative expression and we obtain:

$$
\sum_{h=1}^{n} w_{h} e^{a_{h}+\mathbf{b}_{h}^{\top} \boldsymbol{\mu}+\frac{1}{2} V_{h}+d_{h}^{2} / 2}\left(1+d d_{h}\right)-1=0
$$

So the first order guess of the maximum point is:

$$
\begin{aligned}
d_{\text {guess }} & =\frac{1-\sum_{h=1}^{n} w_{h} e^{a_{h}+\mathbf{b}_{h}^{\top} \boldsymbol{\mu}+\frac{1}{2} V_{h}+d_{h}^{2} / 2}}{\sum_{h=1}^{n} w_{h} e^{a_{h}+\mathbf{b}_{h}^{\top} \boldsymbol{\mu}+\frac{1}{2} V_{h}+d_{h}^{2} / 2} d_{h}} \\
k_{\text {guess }} & =\sqrt{\boldsymbol{\beta}^{\top} V \boldsymbol{\beta}} d_{\text {guess }}+\boldsymbol{\beta}^{\top} \boldsymbol{\mu}+\alpha
\end{aligned}
$$

